\title{
IL-6 Promotes the Proliferation and Immunosuppressive Function of Myeloid-Derived Suppressor Cells via the MAPK Signaling Pathway in Bladder Cancer
}

\author{
Zhong Zheng $\mathbb{D}^{1},{ }^{1}$ Xinyi Zheng $\mathbb{D}^{2}{ }^{2}$ Yiwen Zhu $\mathbb{D}^{3},{ }^{3}$ Zhixian Yao ${ }^{(D},{ }^{1}$ Weiguang Zhao $\mathbb{D},{ }^{1}$

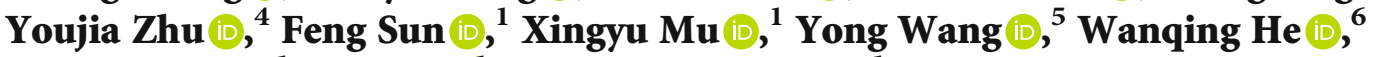

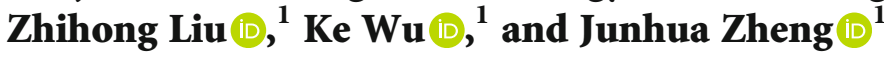 \\ ${ }^{1}$ Department of Urology, Shanghai General Hospital, School of Medicine, Shanghai Jiao Tong University, Shanghai, China \\ ${ }^{2}$ Department of Pharmacy, Huashan Hospital, Fudan University, Shanghai, China \\ ${ }^{3}$ School of Rehabilitation Science, Shanghai University of Traditional Chinese Medicine, Shanghai, China \\ ${ }^{4}$ The Second School of Medicine, Wenzhou Medical University, Wenzhou, China \\ ${ }^{5}$ Department of Urology, Shanghai Jiangqiao Hospital, Jiading Branch, Shanghai General Hospital, Shanghai, China \\ ${ }^{6}$ Student Innovation Center, Shanghai Jiao Tong University, Shanghai, China
}

Correspondence should be addressed to Zhihong Liu; drzhihongliu@sjtu.edu.cn, Ke Wu; doctorwuke@sjtu.edu.cn, and Junhua Zheng; zjhzztg@163.com

Received 27 January 2021; Revised 27 March 2021; Accepted 8 April 2021; Published 24 April 2021

Academic Editor: Fu-Ming Tsai

Copyright (c) 2021 Zhong Zheng et al. This is an open access article distributed under the Creative Commons Attribution License, which permits unrestricted use, distribution, and reproduction in any medium, provided the original work is properly cited.

\begin{abstract}
Muscle-invasive bladder cancer (MIBC) is characterized by a highly complex immune environment, which is not well understood. Interleukin-6 (IL-6) is generated and secreted by multifarious types of cells, including tumor cells. This study was aimed at demonstrating that the levels of IL-6 and the number of myeloid-derived suppressor cells (MDSCs), with a positive correlation between them, increased in MIBC tissues, promoting MIBC cell proliferation, especially in patients with recurrence. In coculture analysis, MDSCs, with the stimulation of IL-6, could significantly lower the proliferation ability of $\mathrm{CD}^{+}$or $\mathrm{CD}^{+} \mathrm{T}$ lymphocytes. Further, this study demonstrated that IL- 6 could upregulate the mitogen-activated protein kinase (MAPK) signaling pathway in MDSCs. The MAPK signaling inhibitor, aloesin, partially reversed the effects of IL- 6 on MDSCs. These data suggested that IL-6 promoted MIBC progression by not only accelerating proliferation but also improving the immune suppression ability of MDSCs through activating the MAPK signaling pathway.
\end{abstract}

\section{Introduction}

Bladder cancer $(\mathrm{BC})$ has the 14th and 9th highest rates of mortality and incidence among all types of cancers [1], accounting for approximately 900,000 newly diagnosed cases each year, with 250,000 deaths occurring during the same time span [2]. Nearly $75 \%$ of patients are diagnosed with non-muscle-invasive BC (NMIBC) and close to $25 \%$ with muscle-invasive $\mathrm{BC}$ (MIBC) [3]. Similar to other solid tumors, MIBC is classified as immunogenic cancer, with numerous tumor-infiltrating lymphocytes (TILs) in the tumor microenvironment (TME) [4]. Besides tumor cells, the TME includes fibroblasts, endothelial cells, mast cells, macrophagocytes (M $\varphi$ ), TILs, and myeloidderived suppressor cells (MDSCs) [5]. In particular, TILs contribute to the adaptive immunological response in opposition to cancer cells and exert anticarcinoma effects in most malignant tumors, including BC, colorectal cancer, 
lung cancer, and melanoma [6]. Nevertheless, the dysregulation of immunosuppressive TME is intensively associated with the development from NMIBC to MIBC [7]. It is, thus, possible that the immunosuppressive functions of TILs in MIBC tumors are prompted, explaining the poor outcomes in MIBC. However, the mechanisms underlying the peculiar pathophysiologic behavior of TILs in MIBC are unclear.

Heterogeneous MDSCs, a cluster of immature myeloidderived cells, are both immunosuppressive and angiogenic. They result from and suppress inflammatory conditions that support tumor growth [8] by stimulating the accumulation of regulatory $\mathrm{T}$ lymphocytes and suppressing responses initiated by cytotoxic $\mathrm{T}$ cells [9]. In patients bearing solid tumors, including $\mathrm{MIBC}$, the interleukin 6 (IL-6) levels are often elevated and inversely associated with prognosis [10]. IL-6 can be generated in a local cytokine niche and is produced by diverse tissues, notably proinflammatory cell types, containing dendritic cells (DCs), $\mathrm{M} \varphi$, MDSCs, T lymphocytes, cancer-associated fibroblasts, and endothelial cells [11]. IL-6 knockdown can affect the immune status, inducing the infiltration of MDSCs. Hence, the mechanisms underlying IL-6-mediated MDSC penetration and functionality should be further explored [12].

Mitogen-activated protein kinases (MAPKs) are serinethreonine protein kinases that modulate a large number of cellular processes, such as innate immunity, survival, apoptosis, differentiation, proliferation, and inflammation [13]. In the wake of tissue damage or pathogen infection, the initiation of pattern recognition receptors in the cell plasma and on the cellular surface of innate immune cells activates a majority of MAPK subfamilies, that is, p38 MAPK, extracellular signal-regulated kinase (ERK), and Jun N-terminal kinase subfamilies. In combination with the upregulation of interferon-regulatory factor as well as nuclear factor- $\kappa \mathrm{B}, \mathrm{MAPK}$ activation leads to the upregulation of a plethora of relevant genes that modulate the immune responses together [13]. The ERK signaling pathway mediates the transition of MDSCs. Its downregulation is correlated with MDSC differentiation. The dysregulation of MAPK signaling pathways promotes the pathogenesis of malignant tumors [14].

This study was aimed at exploring the mechanisms by which IL-6 promotes the immunosuppressive functions and proliferation of MDSCs via the MAPK signaling pathway in MIBC.

\section{Materials and Methods}

2.1. Patient Materials. Malignant lesions and adjacent normal controls were obtained from 200 patients (aged 53-88 years) diagnosed with MIBC who underwent radical cystectomy or transurethral resection of the bladder tumor (TURBT). Specimens were obtained from Shanghai General Hospital, Shanghai, China. The removal of tissues and subsequent analyses were approved by the ethics committee of the hospital and performed after obtaining informed consent from the patients' relatives.
2.2. In Vivo Experiments. C57BL/6 mice (Shanghai Model Organisms Center, China), aged 6-8 weeks, were subcutaneously (s.c.) injected with MB49 cells $\left(2 \times 10^{4}\right)$ and raised under pathogen-free conditions in the Shanghai General Hospital Animal Center. In the IL-6 treatment group, the mice were randomly divided into the experimental group $(n=20)$ and control group $(n=20)$. The grouping conditions in the MDSC transfer group were identical with those in the IL-6 treatment group, but it was launched after the sacrifice of mice on the 30th day in the IL-6 control group owing to the requirement of MDSCs. The $\mathrm{BC}$ tissues of the mice in the IL-6 control group after relevant analysis were subsequently used for MDSC separation and transfer. All the in vivo experiments were launched 10 days (the average number of days for the visible tumor node) after tumor implantation. In the IL-6 treatment group, the mice were intraperitoneally injected with phosphate-buffered saline (PBS) or recombinant murine IL-6 (aa 20-357, $20 \mathrm{ng} / \mathrm{g}$ body weight; carrier-free, R\&D Systems) at 10 a.m. every day. In addition, MDSCs from BC tissues $\left(5 \times 10^{5}\right.$ cells/mouse $)$ mentioned earlier were adoptively transferred to $\mathrm{BC}$ mice via tail vein injection every 7 days, and PBS as a vehicle was administered to the control group. The tumor growth rate was calculated by measuring the estimated tumor volume with a digital caliper every 5 days. The estimated tumor volume was determined using the canonical formula: volume $\left(\mathrm{cm}^{3}\right)=$ $1 / 2 \times(\text { width })^{2} \times$ length. After 30 days, 10 mice randomly extracted from each group were euthanized. The tumor tissues were collected for flow cytometry and MDSC separation for further experiments, including coculture analysis, quantitative reverse transcription polymerase chain reaction (qRT-PCR), and RNA sequencing, under specific conditions. The remaining 10 mice per group were kept for survival analysis. The survival curves for all groups were established using the Kaplan-Meier criteria. The mice were considered expired when the tumor volume reached $2.5 \mathrm{~cm}^{3}$ posttreatment in concordance with the regulations released by the Institutional Animal Care and Use Committee. The animal study was scrutinized and approved by the Institutional Review Board of Shanghai Jiao Tong University.

2.3. Immunohistochemistry. The slices from the formalinfixed paraffin-embedded blocks of MIBC specimens were obtained, and an anti-IL-6 antibody (ab233706, Abcam) was applied for immunostaining to investigate the expression of IL-6. Slices $(4 \mu \mathrm{m})$ of MIBC were loaded on poly-Llysine-coated slides with air drying and deparaffinization. Subsequently, peroxidases were blocked with $5 \%$ hydrogen peroxide in $50 \%$ methyl alcohol at $20^{\circ} \mathrm{C}$ for $20 \mathrm{~min}$. Then, antigen retrieval was performed using a microwave oven for $20 \mathrm{~min}$ in citrate buffer solution. The slices were treated with a monoclonal rabbit anti-IL-6 antibody for $12 \mathrm{~h}$ at $4^{\circ} \mathrm{C}$ after blocking nonspecific binding. They were washed with PBS and treated with biotinylated goat antirabbit IgG (ab150077, Abcam) for $30 \mathrm{~min}$, followed by rinsing with PBS. The immune reactivity was examined 
using the avidin-biotin-peroxidase complex method utilizing VECTASTAIN ABC Kit (PK-4001, Vector). Then, the slices were stained with hematoxylin, dehydrated, cleared, and mounted. The tissues manifesting brown staining in the membrane, nucleus, or cytoplasm were considered positive. The average optical density (AOD) was analyzed using ImageJ software (version 2.0.0, Rawak Software, Inc., Germany) to investigate the correlation between IL-6 levels and MDSCs.

2.4. IL-6 Enzyme-Linked Immunosorbent Assay. The IL-6 enzyme-linked immunosorbent assay (ELISA) was applied according to the product specification (ab178013 for humans and ab100713 for mice, Abcam). Duplicate aliquots of the supernatant were dispersed in the flatbottom 96-well plates. The assay was implemented triply on independent samples.

2.5. Flow Cytometry and MDSC Isolation. Fresh tumor and adjacent tissues were used to prepare single-cell suspensions. The antibodies used for flow cytometry were provided by BioLegend and are listed in Table S1. The cells were examined using a FACSCalibur Flow Cytometer (Becton Dickinson). The cells were stained with carboxyfluorescein succinimidyl ester (CFSE) and analyzed using a flow cytometer (Thermo Fisher) following the manufacturer's protocol. The cells were fixed, permeabilized, and stained with anti-IL-6-FITC (501104, BioLegend) for $30 \mathrm{~min}$ to detect the expression of IL-6. Data were analyzed using FlowJo software (version 10.7.1, Treestar). For MDSC flow cytometric sorting, $1 \times 10^{7}$ cells $/ \mathrm{mL}$ single-cell suspensions made from malignant tissues derived from tumor-bearing mice were applied with conjugated antibodies shown in Table S1 for $20 \mathrm{~min}$ on ice in staining buffer (1\% FBS in PBS). Then, the cells were rinsed with PBS, and the samples were sorted using a BD Influx. The 1.5-drop pure sort mode was selected to obtain cells with high purity. Subsequently, isolated MDSCs were maintained for experiments as follows. The cell culture passage number was no more than 10 for all isolated cells, and three replicates were performed in each passage.

2.6. RT-PCR Analyses of IL6, Arg1, and iNOS. Total RNA was obtained using the RNeasy Mini Kit (27104, Qiagen). Reverse transcription was performed using the TaKaRa RNA PCR kit (RR012A, TaKaRa) following the manufacturer's protocols. The primers were as follows: IL6 (forward $5^{\prime}$-GCTTCC CTCAGGATGCTTGT-3', reverse $5^{\prime}$-ATTAACTGGGG TGCCTGCTC-3'), Arg1 (forward 5'-AGGTTAGAGGC CCAAACTGC- $3^{\prime}$, reverse $5^{\prime}$-TGTCAGCTCCACCGAC TTTC- $3^{\prime}$ ), and iNOS (forward $5^{\prime}$-CTGAAGATACGGAC GGGTCG-3', reverse $5^{\prime}$-CGATGAAGTCGGGGTCGTAG$3^{\prime}$ ) for humans and IL6 (forward $5^{\prime}$-GCCCTCTAGTGGTG CTTGTT-3', reverse $5^{\prime}$-ACTGCAGGCCAGTTACATCC$3^{\prime}$ ), Arg1 (forward 5' -TGCATGCAGTGAAGTGTTGC-3', reverse $5^{\prime}$-AGCCCTAAACCTCCCAATGC- $3^{\prime}$ ), and $i N O S$ (forward $5^{\prime}$-CTGAAGATACGGACGGGTCG-3', reverse $5^{\prime}$-CGATGAAGTCGGGGTCGTAG-3') for mice. The
SYBR Green PCR Master Mix (4309155, Thermo Fisher) and a real-time PCR machine (Applied Biosystems) were used in the reactions. All experiments were performed in triplicate.

2.7. Immune Suppression. Lymphocytes from plasma were isolated by the density-gradient centrifugation using Ficoll-Hypaque solution (Solarbio). $\mathrm{CD}^{+}$microbeads (130-045-201, Miltenyi Biotec) or $\mathrm{CD}^{+}$microbeads (130045-101, Miltenyi Biotec) were used for $\mathrm{T}$ cell sorting. The freshly isolated $\mathrm{CD}^{+} \mathrm{T}$ cells or $\mathrm{CD}^{+} \mathrm{T}$ cells were subsequently cultured in 96-well round-bottomed plates under the conditions that a complete culture medium contained $500 \mathrm{ng} / \mathrm{mL}$ soluble anti-CD28 (T9-577-T100, American Research Products) and $500 \mathrm{ng} / \mathrm{mL}$ soluble or immobilized anti-CD3 (T1P1D4*C6, American Research Products) for $\mathrm{T}$ cell stimulation and activation. The $\mathrm{CD}^{+}$or $\mathrm{CD}^{+} \mathrm{T}$ cell proliferation was evaluated using the CFSE Cell Division Tracker Kit (423801, BioLegend). MDSCs were added to the coculture at different ratios to $\mathrm{CD}^{+} \mathrm{T}$ cells or $\mathrm{CD}^{+}{ }^{+} \mathrm{T}$ cells for $72 \mathrm{~h}\left(\mathrm{CD} 8^{+} \mathrm{T}\right.$ cells $/ \mathrm{CD} 4^{+}$ $\mathrm{T}$ cells to $\mathrm{MDSCs}=4: 1$ and $2: 1), 48 \mathrm{~h}$ after $\mathrm{T}$ cell stimulation. The cells were then collected and treated with the $\mathrm{APC}-\mathrm{CD}^{+} \mathrm{T}$ cell antibody $(344722$ for humans and 100712 for mice, BioLegend) or $\mathrm{APC}-\mathrm{CD} 4^{+} \mathrm{T}$ cells (357408 for humans and 100412 for mice, BioLegend) prior to flow cytometry. The cell culture passage number was no more than 10 for all isolated cells, and three replicates were performed in each passage. All experiments were performed in triplicate.

2.8. Western Blot Analysis. The cells were completely lysed in proteinase-containing radioimmunoprecipitation assay buffer inhibitor (4693132001, Complete Roche). Protein quantification was applied using the protein assay reagent (5000001, Bio-Rad). Further, $10 \mu \mathrm{g}$ of total protein was used for subsequent western blot (WB) analysis. The lysates were electrophoresed and transferred to Hybond nitrocellulose paper (FFN08, Beyotime). The nonspecific binding was blocked with 5\% skimmed milk in PBS with 0.1\% Tween-20 (P0220, Beyotime). For target protein examination, the blots were treated with the primary anti-human or anti-mouse antibodies at $4^{\circ} \mathrm{C}$ overnight. The blots were rinsed with PBST and treated with the HRP-conjugated goat anti-rabbit IgG antibody (ab6702, Abcam) for $60 \mathrm{~min}$ at $20^{\circ} \mathrm{C}$. The target proteins were detected using the ECL WB substrate kit (ab65623, Abcam), and blots were applied to Amersham Imager 600 (GE Healthcare Life Sciences). The densitometry readings/intensity ratio of each band was analyzed by using Image J software (version 2.0.0, Rawak Software, Inc. Germany). All experiments were performed in triplicate. All relevant antibodies are listed in Table S2.

2.9. RNA Sequencing. Total RNA was obtained and enriched for mRNA using MicroPoly A Purist (AM1919, Ambion). The RNA quantity and quality were assessed using Bioanalyzer 2100 (Agilent Technologies). RNA sequencing was performed using the Illumina HiSeq 2000 


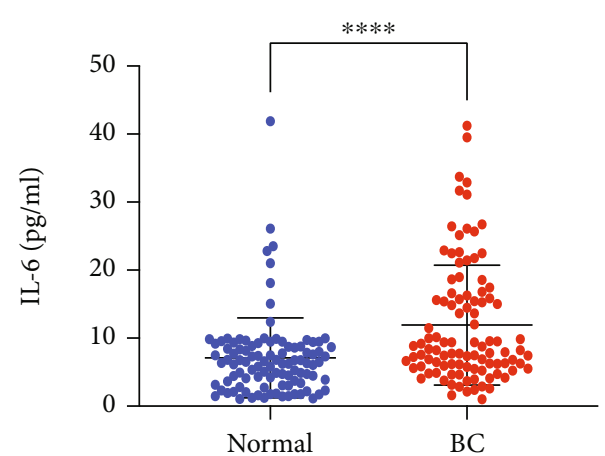

(a)

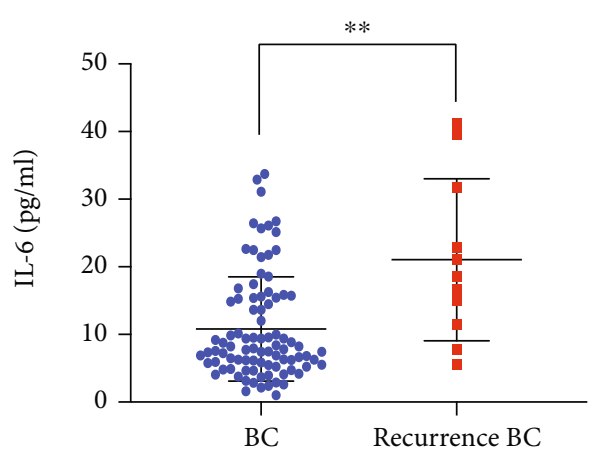

(c)

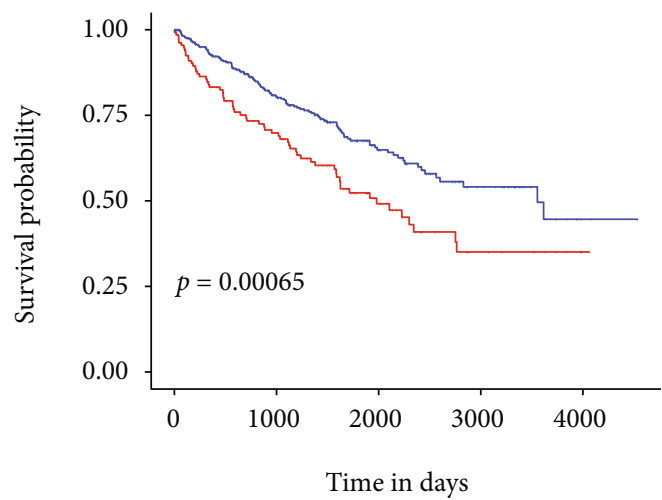

Expression Level

- High expression $(n=134)$

— Low/medium expression $(n=397)$

(e)

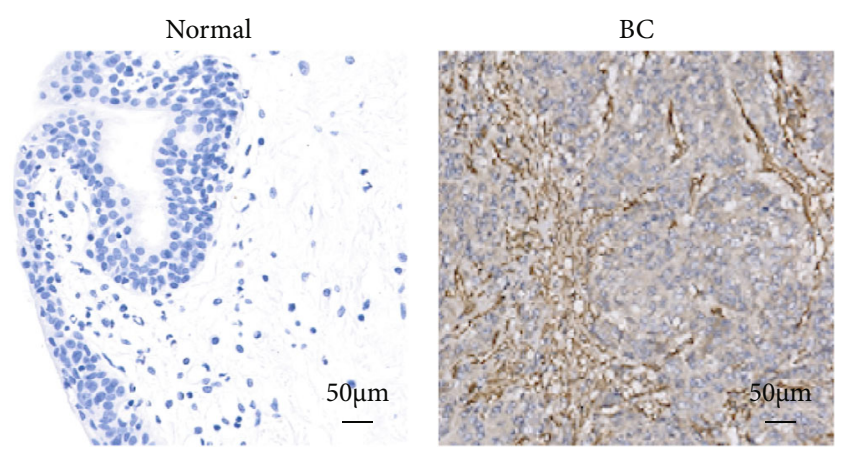

(b)
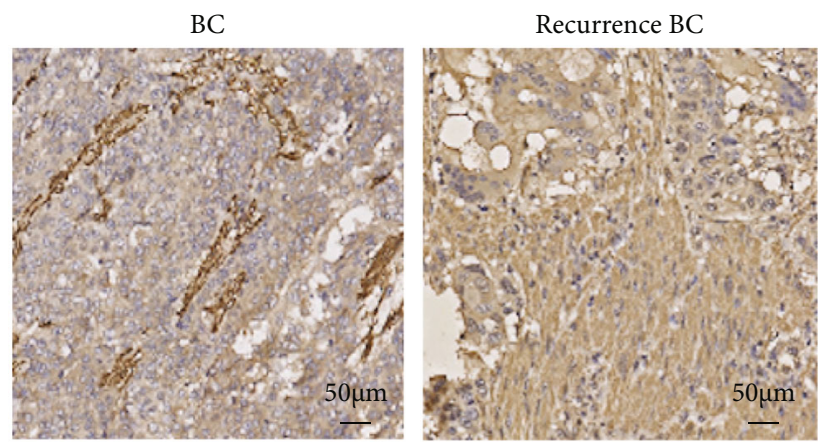

(d)

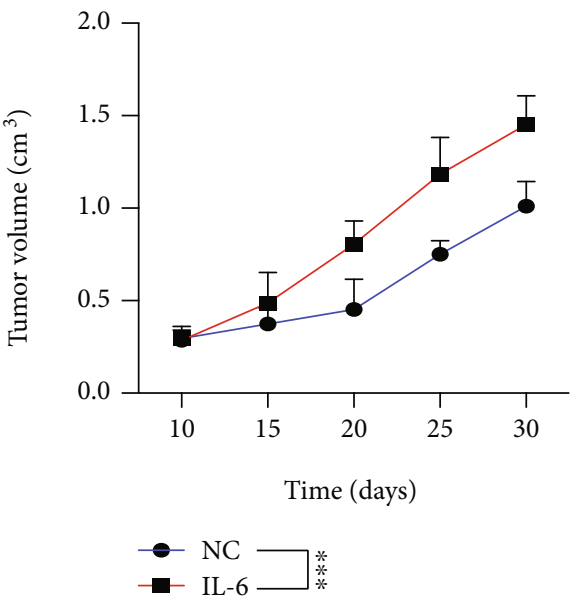

(f)

FIGURe 1: Continued. 


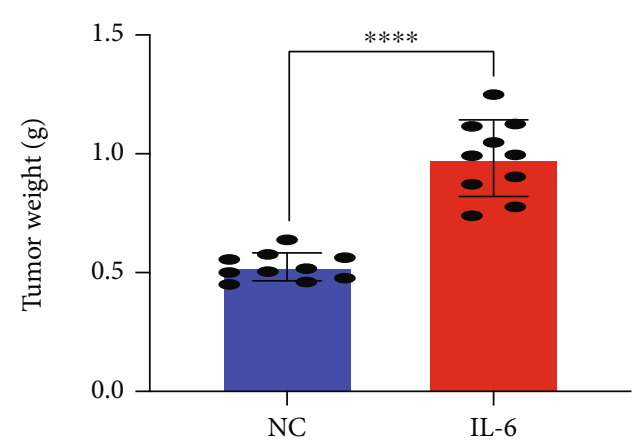

(g)

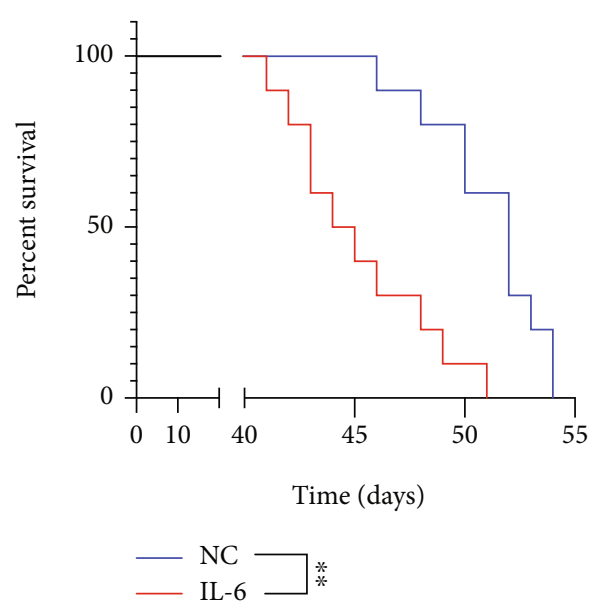

(h)

FIGURE 1: Interleukin 6 (IL-6) levels increased in muscle-invasive bladder cancer (MIBC) and promoted cancer progression. (a) IL-6 levels in MIBC tissues and normal urothelium determined by the enzyme-linked immunosorbent assay (ELISA). (b) Immunochemistry results for IL-6 in MIBC tissues and normal urothelium. (c) IL-6 levels in MIBC tissues and recurrent MIBC determined by ELISA. (d) Immunochemistry results for IL-6 in MIBC tissues and recurrent MIBC. (e) Prognosis of patients with high or low IL-6 expression according to TCGA database. (f) MIBC tumor volume with or without IL-6 treatment $(n=10)$. (g) Tumor weight with or without IL-6 treatment $(n=10)$. (h) Survival time for mice with MIBC with or without IL-6 treatment $(n=10)$. Mean $\pm \mathrm{SD},{ }^{* *} P<0.01,{ }^{* * *} P<0.005$, and ${ }^{* * * *} P<0.001$.

platform (Illumina) for each sample. Digital gene expression profiles were used to analyze the differential gene expression between groups. The pathway enrichment was analyzed to further elucidate the cellular mechanisms.

2.10. Statistical Analysis. The results were expressed as means \pm standard deviation. Analyses of variance and unpaired or paired-sample Student's $t$-tests were used for in vitro and in vivo experiments.

\section{Results}

3.1. IL-6 Levels Were Elevated in MIBC Cells and Associated with Cancer Progression. MIBC tissues $(n=200)$ were collected and compared with the normal urothelium $(n=200)$, which included 11 recurrent MIBC tissues to test the IL-6 expression level in MIBC cells. ELISA showed that the IL-6 level was higher in MIBC tissues than in adjacent noncancer tissues (Figure 1(a)). Immunochemistry showed that IL-6 staining was more obvious in MIBC tissues than in adjacent tissues (Figure 1(b)). Also, IL-6 levels were compared between recurrent and primary MIBC. ELISA showed that the IL-6 levels were higher in recurrent MIBC tissues than in primary MIBC tissues. Immunochemistry also showed that IL-6 staining was more obvious in recurrent MIBC tissues than in primary MIBC tissues (Figures 1(c) and 1(d)). According to TCGA database, high IL-6 expression was associated with a worse prognosis in patients with MIBC than in patients with low IL-6 levels (Figure 1(e)). In vivo, MIBC mouse models were treated with IL-6 for the analyses of tumor growth rates and tumor weights. IL- 6 promoted MIBC growth
(Figures 1(f)-1(g)). Mice with MIBC treated with IL-6 showed shorter survival times compared with untreated control mice (Figure 1(h)). These results showed that IL6 levels were elevated in MIBC tissues, especially in cases of recurrence, and promoted MIBC progression by accelerating proliferation.

3.2. Number of MDSCs Increased in MIBC Tissues and Promoted Cancer Progression. MDSCs are a heterogeneous cluster of immune cells deriving from the myeloid lineage with an immune suppression role in MIBC. MDSCs were identified as $\mathrm{CD}^{+} 5^{+} \mathrm{CD} 11 \mathrm{~b}^{+} \mathrm{HLA}-\mathrm{DR}^{-} \mathrm{CD} 33^{+}$by flow cytometry as previously studied [15], and the frequency of MDSCs was found to be higher in MIBC tissues than in the normal urothelium (Figures 2(a) and 2(b)). The increase in the number of MDSCs was greater in recurrent MIBC than in primary MIBC tissues (Figures 2(c) and 2(d)). In vivo, MDSCs were adoptively transferred into mice with MIBC, and cancer progression was evaluated. MDSCs increased tumor growth rates (Figure 2(e)). The tumor weights were higher in the MDSC-treated group compared with the control group (Figure 2(f)). Based on survival time, MDSC transfer in the MIBC mouse model was related to a worse prognosis (Figure 2(g)). Taken together, MDSCs increased in MIBC tissues and promoted cancer progression.

3.3. IL-6 Promoted MDSC Proliferation in MIBC Tissues. IL-6 is closely related to several immune responses, including MDSC-induced immune suppression. A total of 100 MIBC tissues were collected to explore the relationship between IL-6 and MDSCs. Immunohistochemistry and 


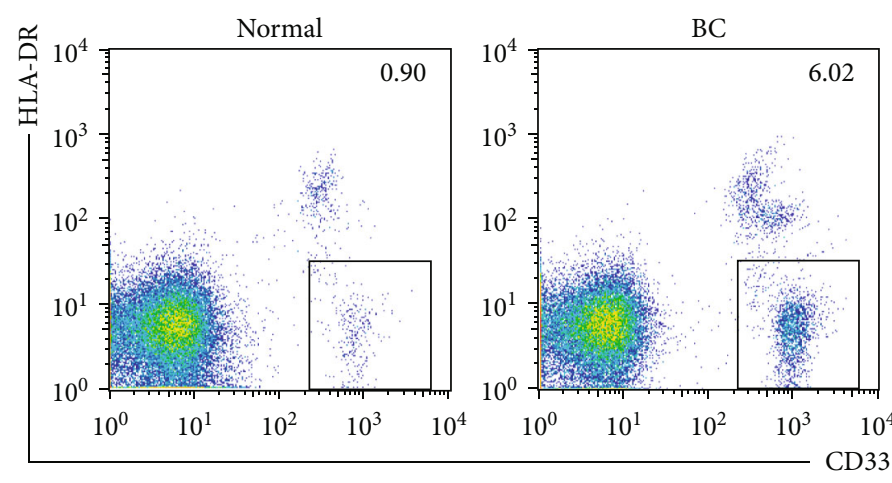

(a)

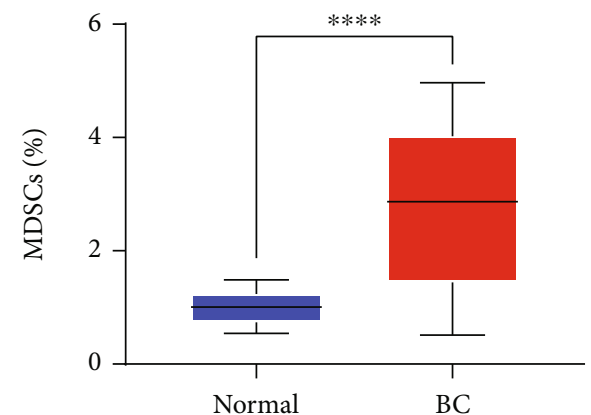

(b)
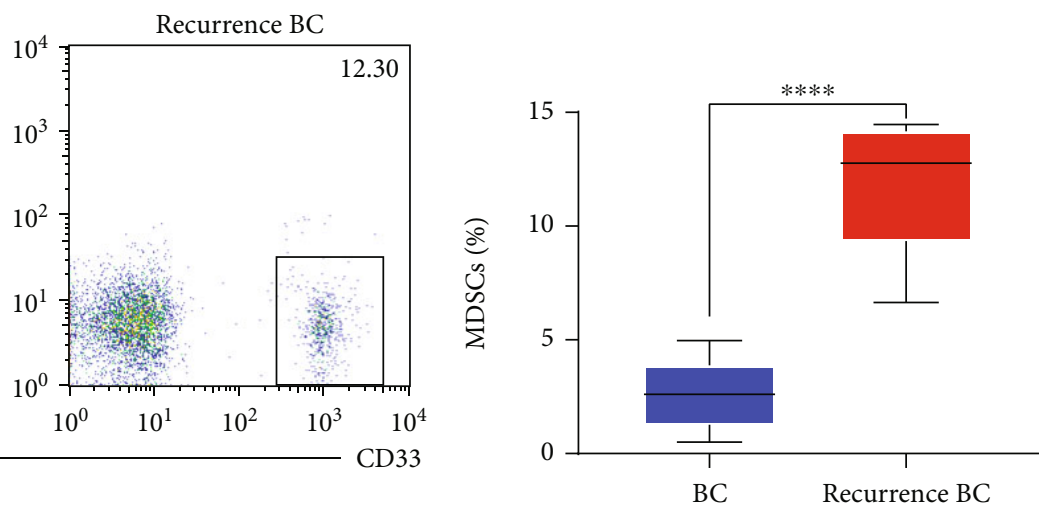

(d)
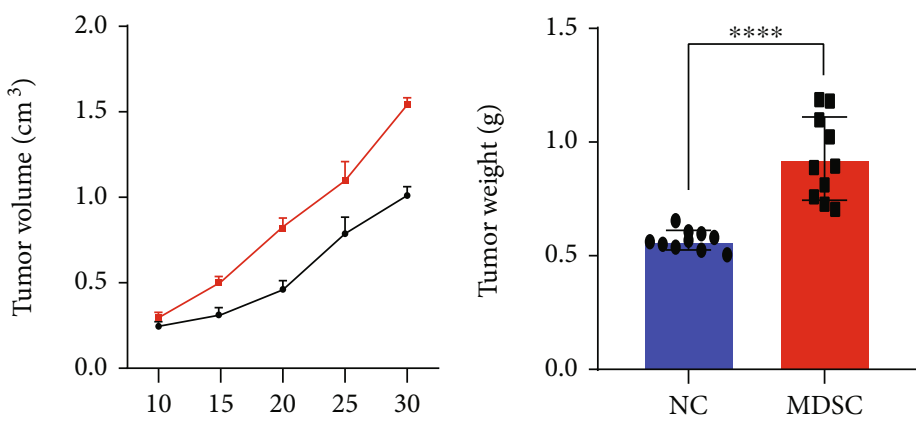

Time (days)

$\rightarrow \mathrm{NC}-{ }_{*}^{*}$

(e)

(f)

Figure 2: Continued. 


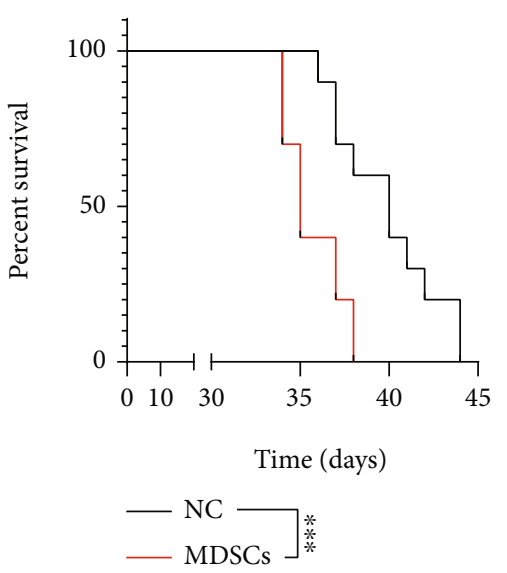

(g)

FIgURE 2: Myeloid-derived suppressor cells (MDSCs) increased in MIBC tissues and promoted cancer progression. (a, b) Flow cytometry and statistical analysis of the proportion of MDSCs in MIBC tissues and normal urothelium from 200 patients. (c, d) Flow cytometry and statistical analysis of the proportion of MDSCs in recurrent MIBC tissues and in MIBC tissues. (e) Tumor growth rates in a MIBC mouse model with or without MDSC treatment $(n=10)$. (f) Tumor weight in a MIBC mouse model with or without MDSC treatment $(n=10)$. (g) Survival time of a MIBC mouse model with or without MDSC treatment $(n=10)$. Mean $\pm \operatorname{SD},{ }^{* * *} P<0.005$, and ${ }^{* * * *} P$ $<0.001$.

flow cytometry were used to evaluate IL-6 levels, and flow cytometry was used to quantify MDSCs. The IL-6 levels were positively related to MDSCs (Figures 3(a) and 3(b)). In vitro, MDSCs were sorted from mouse MIBC tissues and treated with or without IL-6. As summarized in Figure 3(c), the CCK-8 assay showed that the rate of proliferation was higher in IL-6-treated MDSCs compared with the control cells (Figure 3(d)). CFSE further demonstrated that IL-6 promoted the proliferation of MDSCs (Figure 3(e)). The flow cytometry images also showed that IL-6 increased the proportion of MDSCs in both humans and mice (Figures 3(f) and 3(g)). These results showed that IL- 6 accelerated MDSC accumulation by promoting proliferation.

3.4. IL-6 Improved the Immune-Suppressive Activity of MDSCs. MDSCs were sorted from human and mouse MIBC tissues to explore whether IL-6 influenced the immune-suppressive effects of MDSCs. MDSCs treated with or without IL-6 were collected and prepared for follow-up experiments (Figure 4(a)). RT-PCR showed that IL-6 treatment increased Arg1 and iNOS levels (Figures 4(b)4(e)). MDSCs were cocultured with $\mathrm{CD}^{+} \mathrm{T}$ cells or $\mathrm{CD} 4^{+} \mathrm{T}$ cells at different ratios (T cells : MDSCs $=1: 4$ or $1: 2$, respectively). IL-6-treated MDSCs significantly impeded the proliferation of $\mathrm{CD}^{+} \mathrm{T}$ cells and $\mathrm{CD}^{+} \mathrm{T}$ cells (Figures 4(f)-4(i)). Accordingly, IL-6 promoted the proliferation of MDSCs and immune-suppressive activity.

3.5. IL-6 Activated the MAPK Signaling Pathway in MDSCs. MDSCs were collected from human and mouse MIBC tissues to explore the specific mechanism underlying the effect of IL- 6 on MDSCs. MDSCs were treated with IL-6 for $48 \mathrm{~h}$ and used for RNA sequencing
(Figures 5(a) and 5(b)). Differentially expressed genes were enriched for several signaling pathways, especially the MAPK signaling pathway (Figures $5(\mathrm{c})$ and $5(\mathrm{~d})$ ). To confirm this result, WB analysis was performed to evaluate the key proteins with or without phosphorylation in the MAPK signaling pathway (MEK2, ERK, and MNK1). IL6 treatment induced the phosphorylation of MEK2, ERK, and MNK1 in MDSCs from both human and mouse MIBC tissues (Figures 5(e)-5(i)). These results indicated that IL-6 promoted the proliferation and immunesuppressive effect of MDSCs by upregulating the MAPK signaling pathway.

3.6. IL-6 Promoted MDSC Proliferation and ImmuneSuppressive Effects by Activating the MAPK Signaling Pathway. Aloesin was used to block the activation of the MAPK signaling pathway so as to confirm the role of the MAPK subfamilies in MDSC proliferation and immune-suppressive effects. The CCK-8 assay and flow cytometry results showed that aloesin partially attenuated the IL-6-induced increase in proliferation (Figures 6(a) and 6(b)). RT-PCR showed that IL-6 treatment increased Arg1 and iNOS levels, while IL- 6 could reverse this phenomenon (Figures 6(c)-6(f)). MDSCs were cocultured with $\mathrm{CD}^{+} \mathrm{T}$ cells or $\mathrm{CD}^{+} \mathrm{T}$ cells at specific ratios (MDSCs : T cells = $1: 4$ ). IL-6-treated MDSCs significantly impeded the proliferation of $\mathrm{CD}^{+} \mathrm{T}$ cells and $\mathrm{CD} 4^{+} \mathrm{T}$ cells, which was partially reversed by aloesin (Figures $6(\mathrm{~g})-6(\mathrm{j})$ ). These results elucidated that IL- 6 promoted the proliferation of MDSCs and immune-suppressive effects, and these effects could be reversed by targeting the MAPK signaling pathway. Taken together, IL-6 promoted the proliferation and immunosuppressive function of MDSCs via the MAPK signaling pathway in MIBC (Figure 7). 


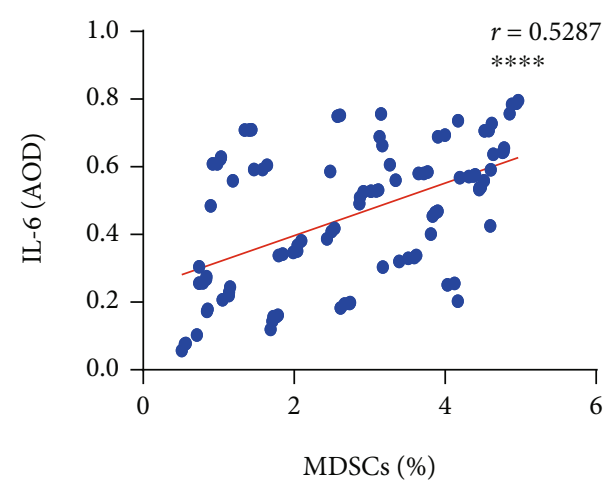

(a)

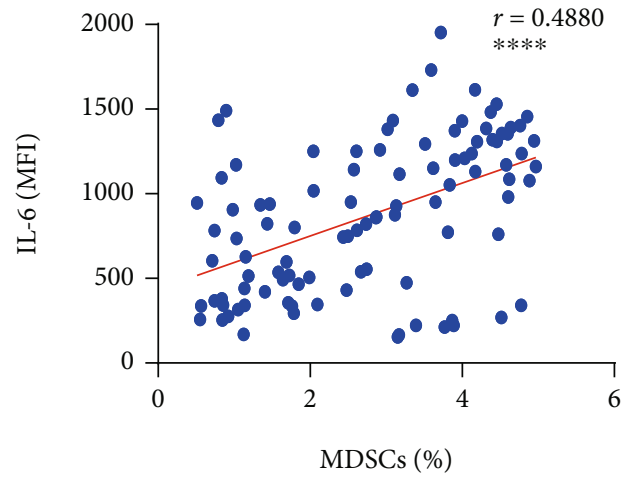

(b)
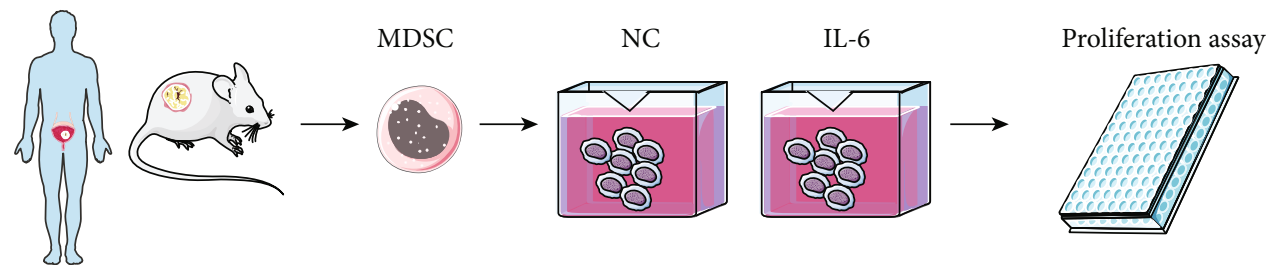

(c)

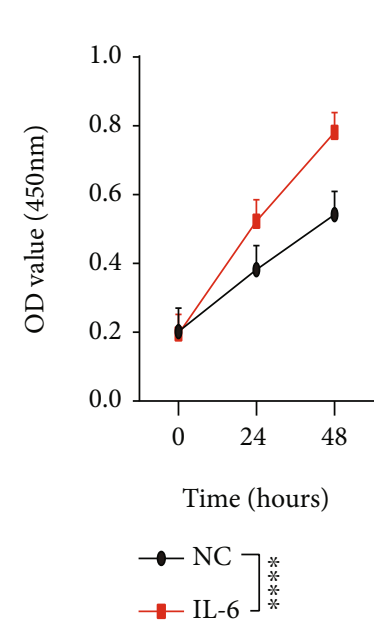

(d)
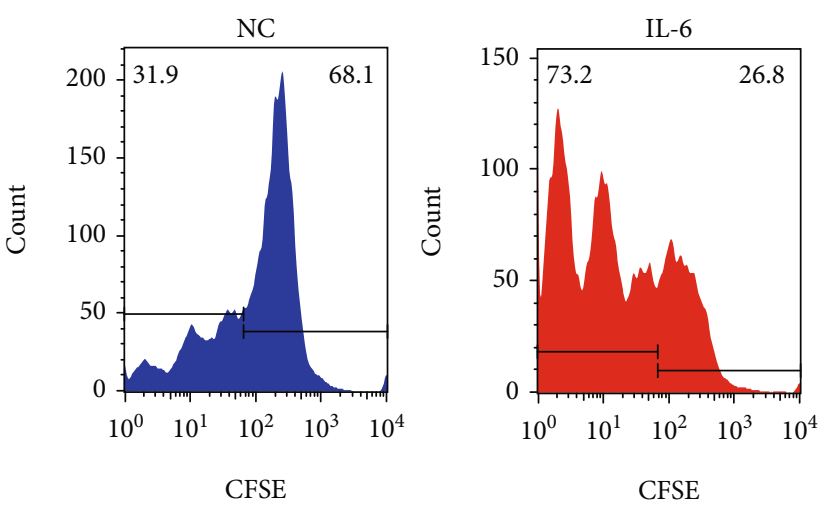

(e)

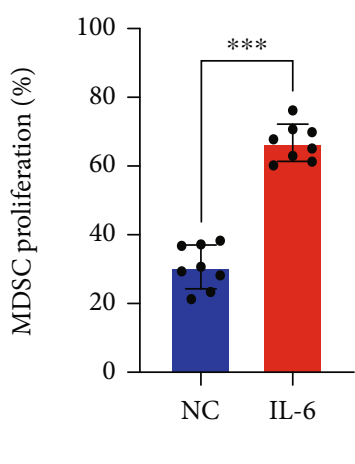

Figure 3: Continued. 

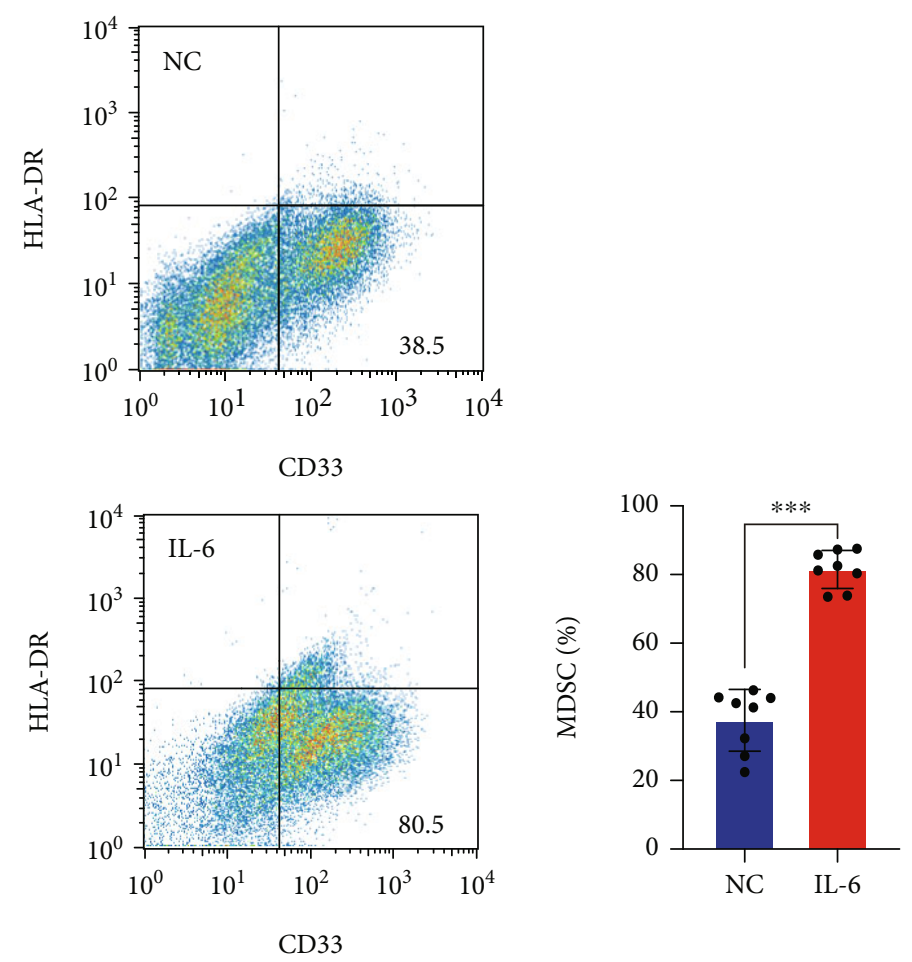

(f)
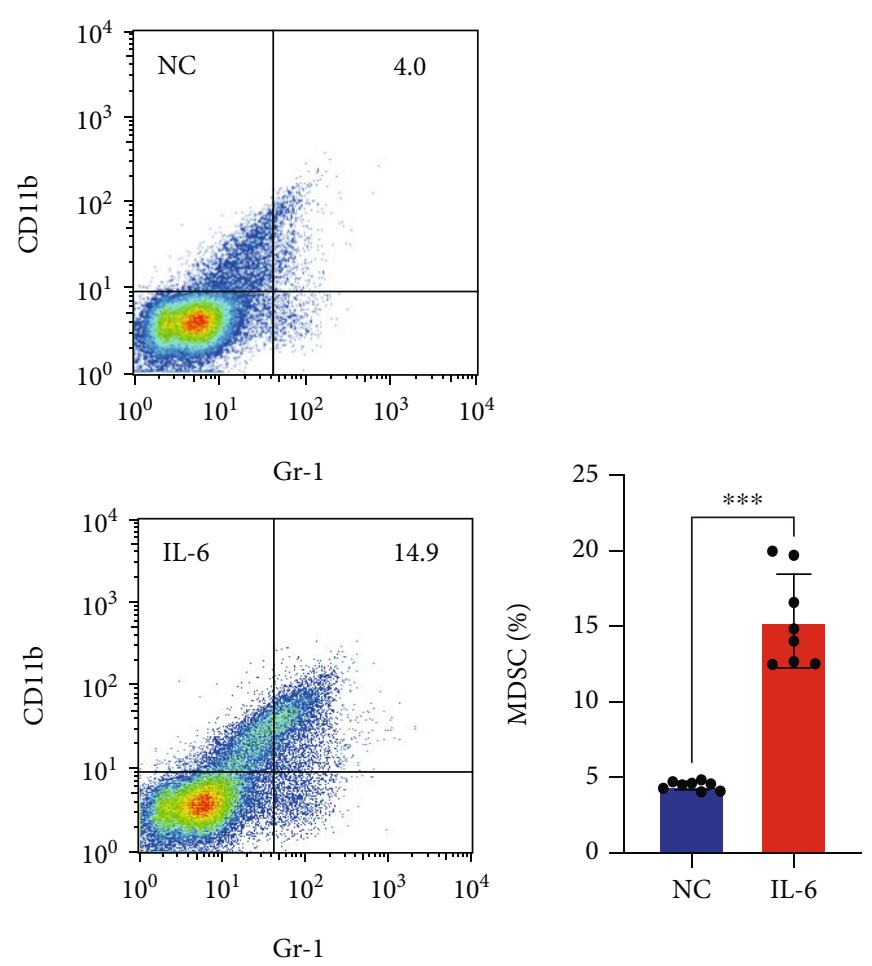

(g)

FIgURE 3: IL-6 promoted MDSC proliferation in MIBC tissues. (a, b) Correlation between IL-6 (AOD and MFI) and MDSCs in MIBC tissues. (c) Experimental design of MDSC extraction and IL-6 treatment. (d) CCK-8 assays were used to test MDSCs with or without IL-6 treatment. (e) MDSC proliferation rates were evaluated according to fluorescence attenuation. (f, g) Flow cytometry images and statistical analysis of MDSCs from humans and mice with or without IL-6 treatment. Mean \pm SD, ${ }^{* * *} P<0.005$, and ${ }^{* * * *} P<0.001$. AOD: average optical density; MFI: median fluorescence intensity. 

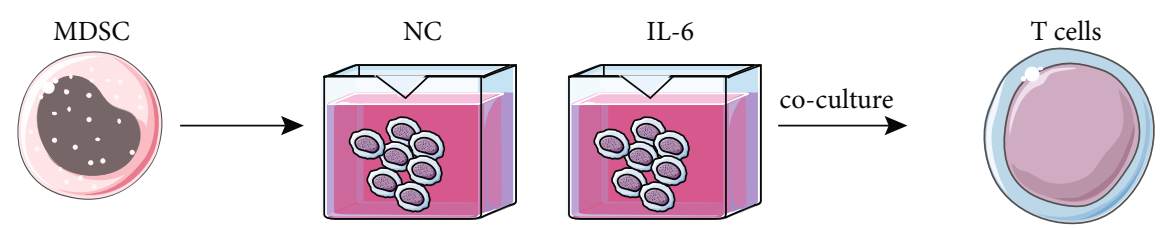

(a)

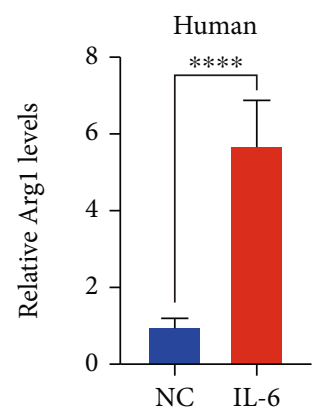

(b)

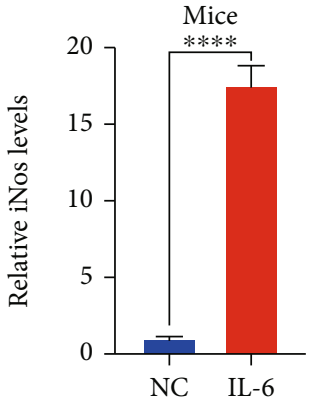

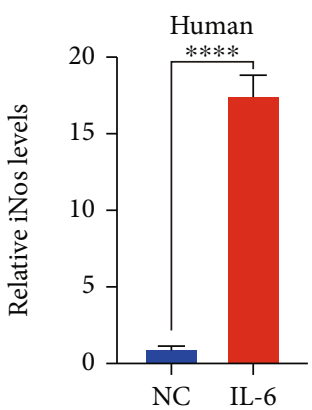

(c)

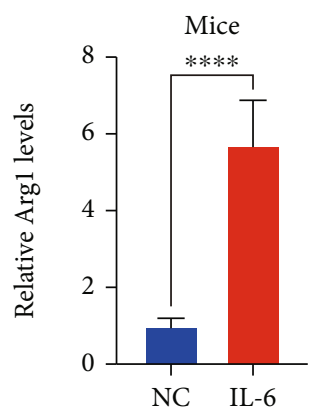

(d)

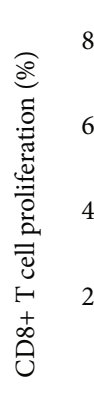

Human

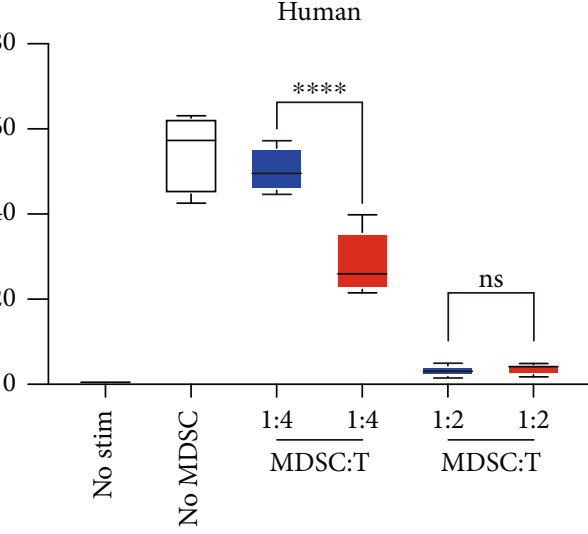

- WT MDSC

MDSC+IL-6

FIgUre 4: Continued. 


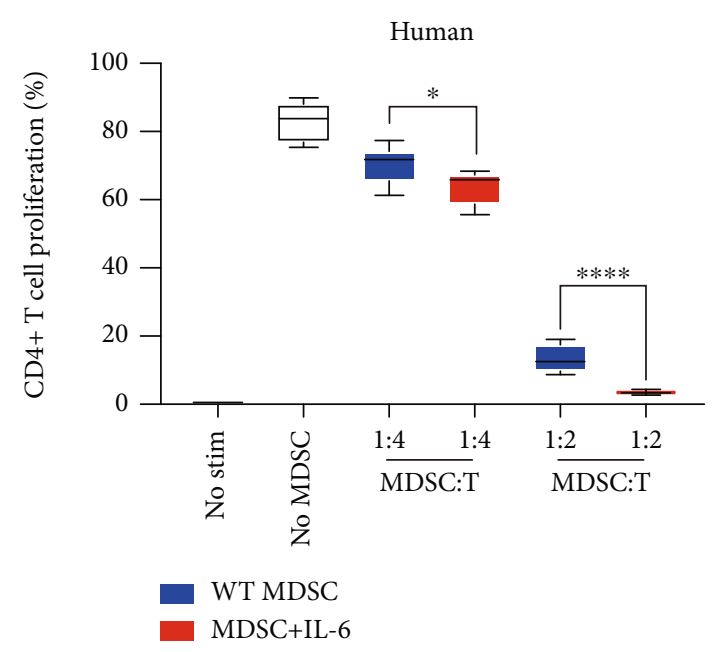

(g)

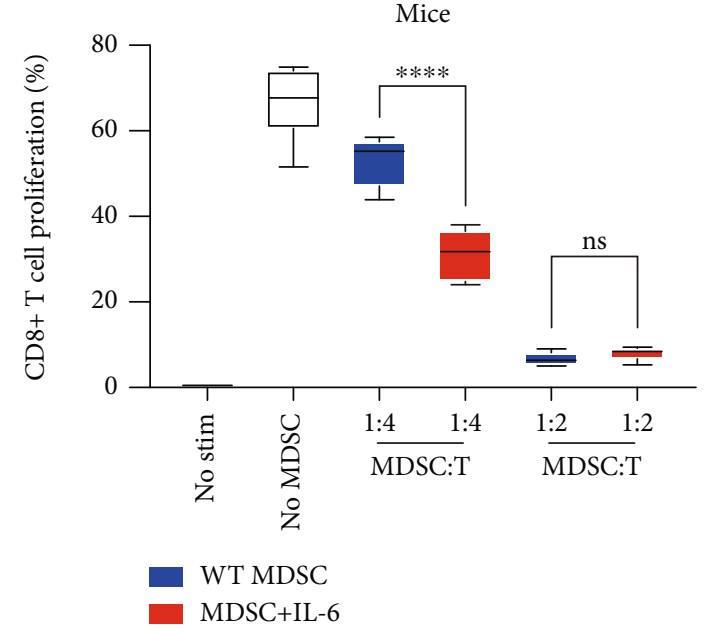

(h)

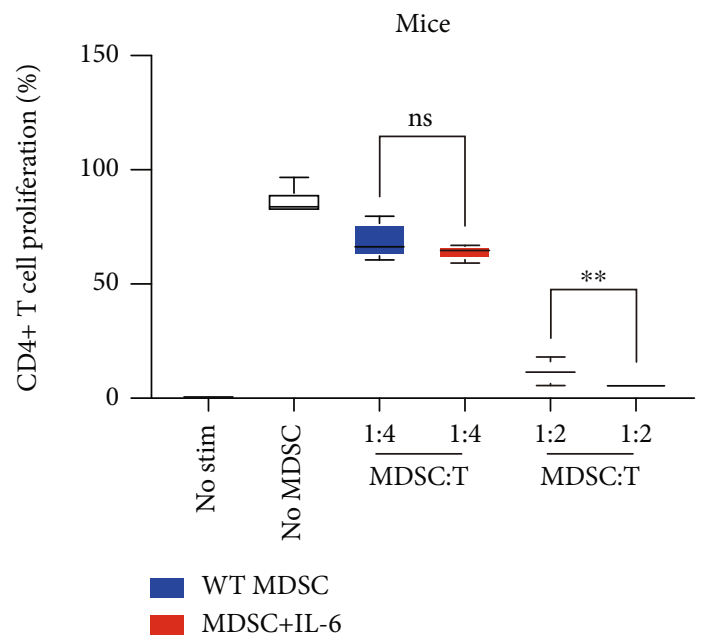

(i)

FIGURE 4: IL-6 improved the immune-suppressive effects of MDSCs. (a) Experimental diagram of MDSC extraction and IL-6 treatment. (b-e) RT-PCR was used to evaluate Arg1 and iNOS levels in MDSCs with or without IL-6 treatment. (f-i) Summary of the suppressive effect of MDSCs by coculture with CD8+ T cells and CD4+ T cells at $1: 4$ and $1: 2$ ratios. Mean $\pm \mathrm{SD},{ }^{*} P<0.05,{ }^{* *} P<0.01,{ }^{* * *} P<0.005$, and ${ }^{* * * *}$ $P<0.001$. ns: no statistical significance.

\section{Discussion}

IL-6 is highly pleiotropic and has a wide range of functions in various diseases [16]. The TME of most malignant lesions shows immune cell enrichment with immunosuppressive phenotypes, often via the immunomodulatory activities of IL-6-family cytokines. The members of this family have been identified as diagnostic or prognostic biomarkers of the response to therapy and disease activity in various cancers [17]. Aberrant IL-6 family overexpression and downstream receptor signaling pathway stimulation are frequent cytokine events in carcinomas and are often correlated with poor clinical outcomes [18]. The tumorigenesis-promoting effects of IL-6 cytokine family members are mediated via direct effects on proliferation, survival, migration, invasion, and metastasis in cancer cells and indirect effects on immunosuppression, modulation of inflammation, and angiogenesis in the interstitial stromal cells, which form the local TME [19]. IL-6 is expressed in numerous BC cell lines and is more abundant in the specimens of patients with MIBC than those with NMIBC [20]. Both BC cell lines and primary BC tissues express IL-6 and IL-6 receptor protein [21]. IL-6 clinically serves as an alternative marker of host immunity in patients with BC [22]. The canonical activation of immunity in BC elicits the production of IL-6, leading to BC-associated immune malfunction [23]. Typically, serum IL-6 level is significantly higher in higher-grade patients than in low-grade patients; furthermore, it is linked to patients with recurrent BC [24]. Patients with elevated IL- 6 levels have a high risk of distant metastases and lymphatic metastases [25]. 


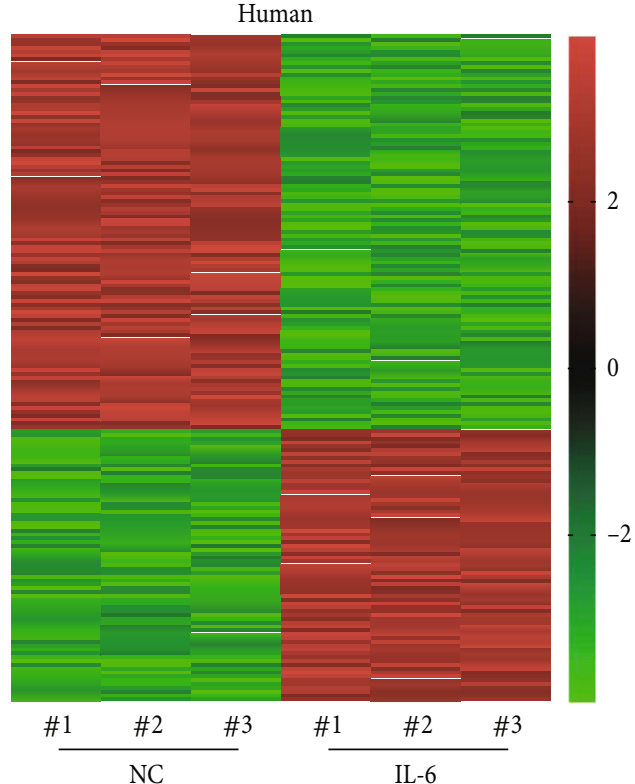

(a)

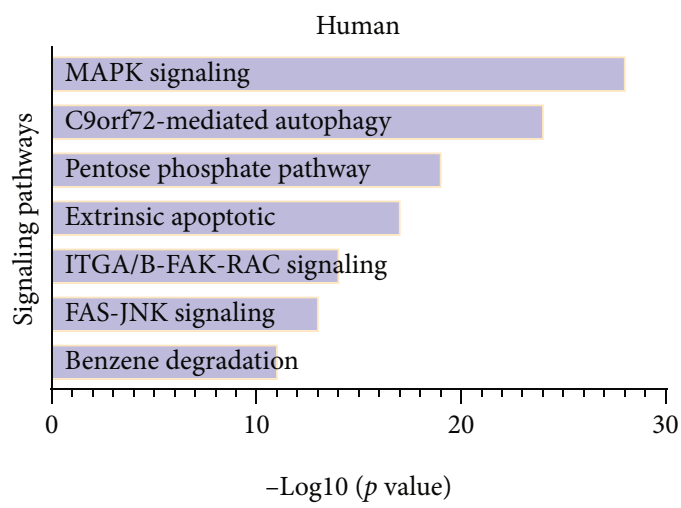

(c)

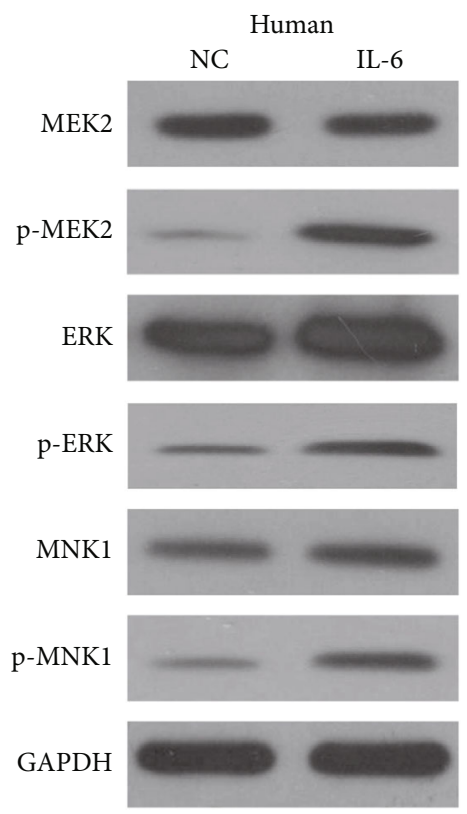

(e)

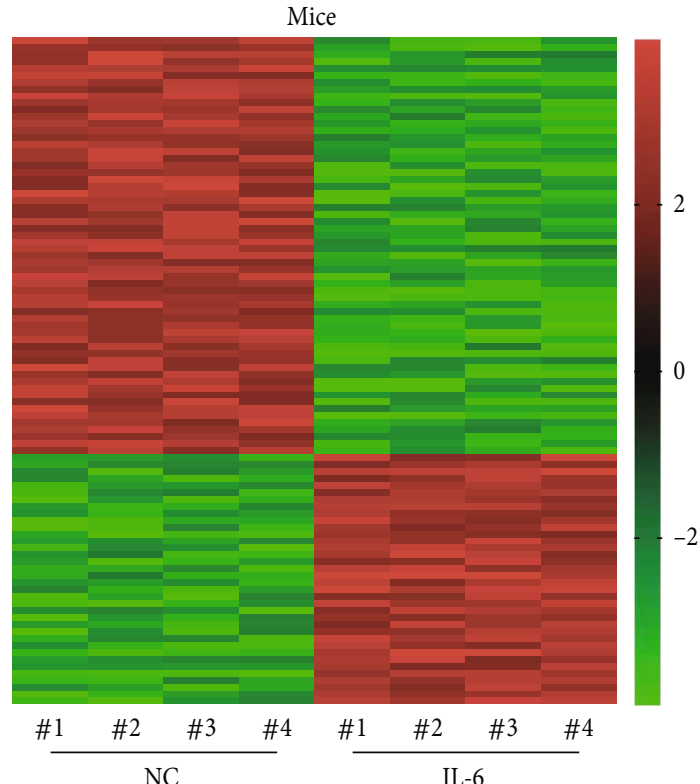

(b)

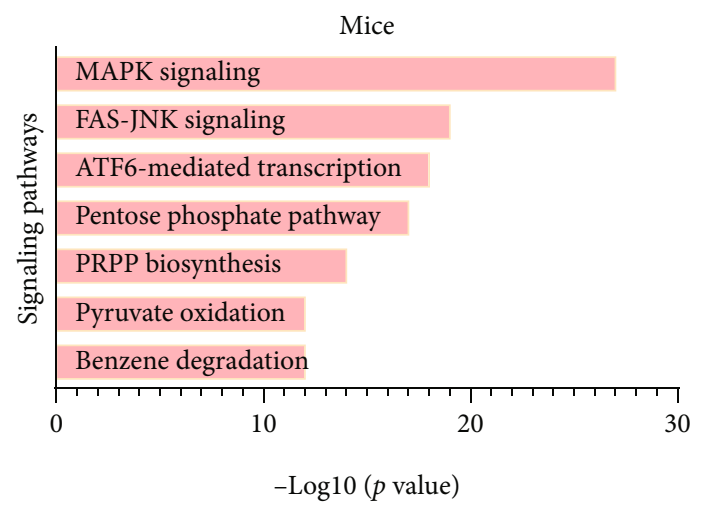

(d)

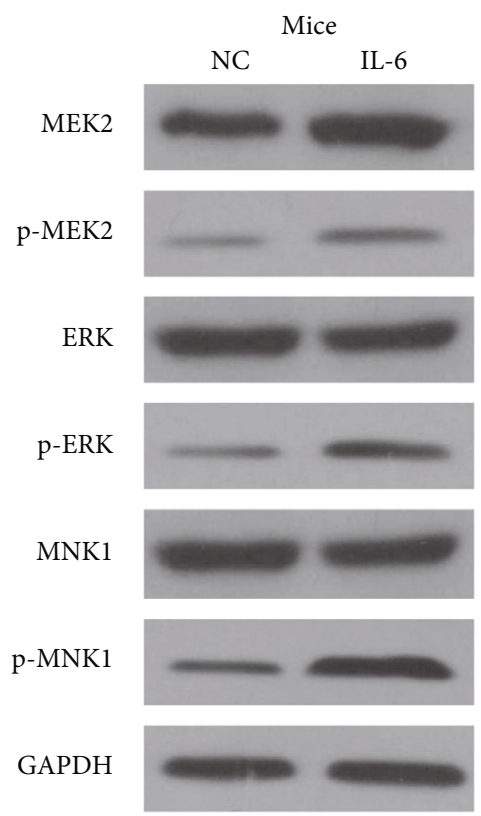

(f)

FIgure 5: Continued. 


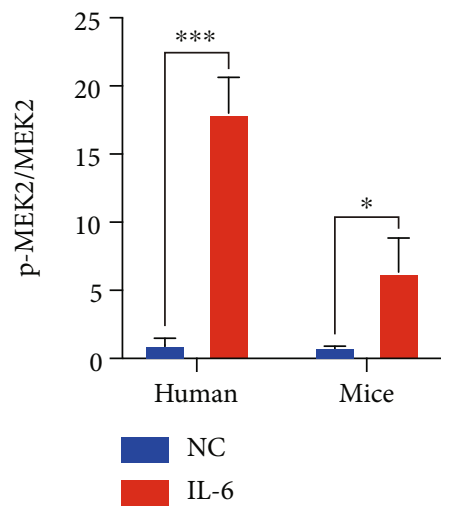

(g)

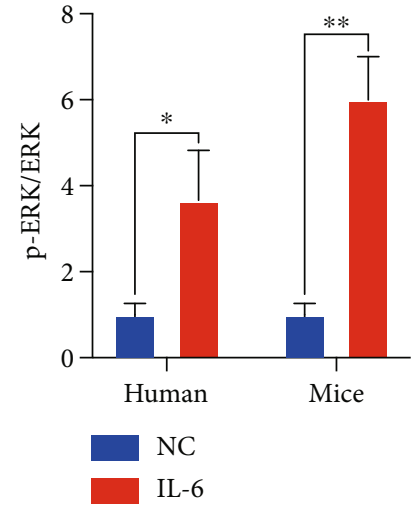

(h)

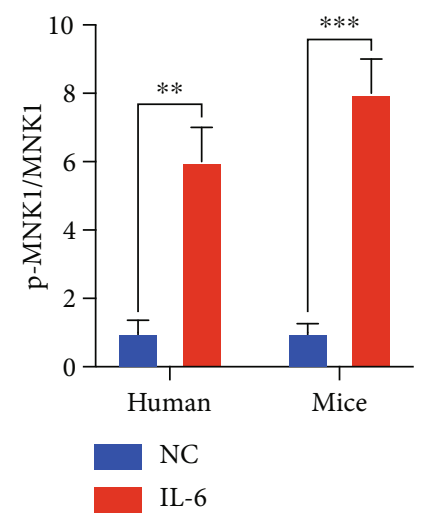

(i)

FIGURE 5: IL-6 activated the mitogen-activated protein kinase (MAPK) signaling pathway in MDSCs. (a, b) Heat map of RNA sequencing results to evaluate differentially expressed genes in MDSCs with or without IL-6 treatment. (c, d) Differentially expressed genes were enriched for several signaling pathways. (e, f) Phosphorylation of critical proteins in the MAPK signaling pathway (MEK2, ERK, and MNK1) was evaluated by western blotting. (g-i) Intensity ratio of each band. Mean $\pm \mathrm{SD},{ }^{*} P<0.05,{ }^{* *} P<0.01$, and ${ }^{* * *} P<0.005$.

Among IL-6 cytokine family members, IL-6 has the most well-defined effect on the TME by prompting chronic inflammatory responses, regulating tumor vasculogenesis and the outgrowth of heterogeneous cells, and preventing Th1 cell-mediated antitumor immunocytotoxicity [26]. These protumorigenic effects are mediated by the recruitment, retention, activation, and function of TILs and reflect the various innate and adaptive immune responses regulated by IL- 6 in immunity and chronic inflammatory diseases [27]. In melanoma, however, IL-6 exerts antitumor effects in the early stages of disease progression and later promotes tumor angiogenesis and the recruitment of immunosuppressive myeloid cells, such as MDSCs, to the TME [28]. Additionally, the tumorpromoting effects of IL-6 involve increases in MDSCs and Th17 cells, suppression of DCs and cytotoxic T cells, and phenotypic switching of tumor-associated macrophages (TAM) from an antitumor M1 phenotype to an immunosuppressive M2 phenotype [26]. In gastric cancer, serum-derived IL-6 activates and induces MDSCs, which express arginase 1, via the PI3K-Akt signaling pathway, thereby suppressing cytotoxic $T$ cell functionality [29]. In hepatocellular cancer, the interaction between MDSCs and IL- 6 promoted the generation of a chemoresistant phenotype [30]. However, in BC, low IL-6 levels could inhibit the activation of STAT3, promoting the formation of MDSCs [31]. The IL-6-induced experiments were simply performed on MDSCs in the serum of patients with BC. The serum MDSCs might not completely represent MDSCs in the TME of BC where elusive cell-cell communications are abundant, and MDSCs can be immunomodulated and undergo various alterations. The present analysis showed that IL- 6 could stimulate the proliferation of MDSCs from the BC microenvironment in a time- and dose-dependent manner. Also, it indicated that further analysis, such as dose-dependent experiments for IL-6induced MDSC alteration, should be implemented and more details should be elucidated.

MDSCs are primary inhibitors of efficient antitumor immunity as well as $\mathrm{T}$ cell proliferation and immune activity [32]. This study found that the proliferation of MDSCs correlated with increasing levels of IL-6; mice with high levels of IL-6 had increased frequencies of MDSCs. Patients with high frequencies of MDSCs had poorer overall survival compared 


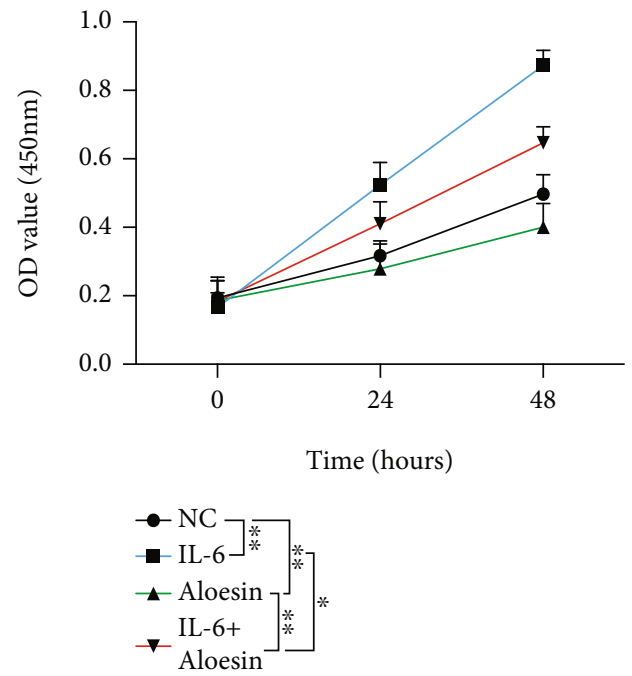

(a)

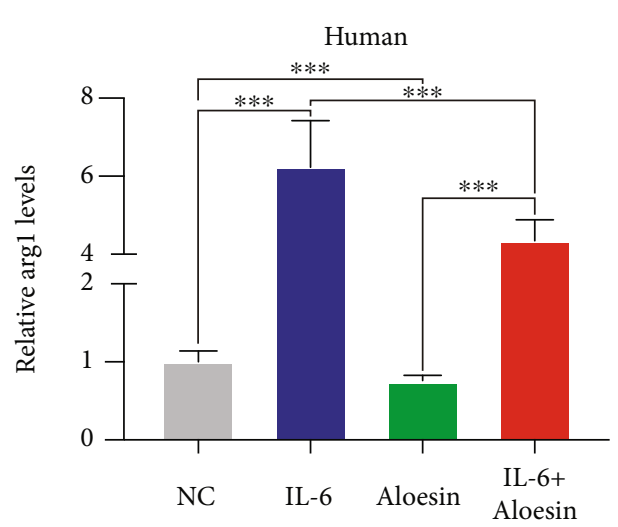

(c)

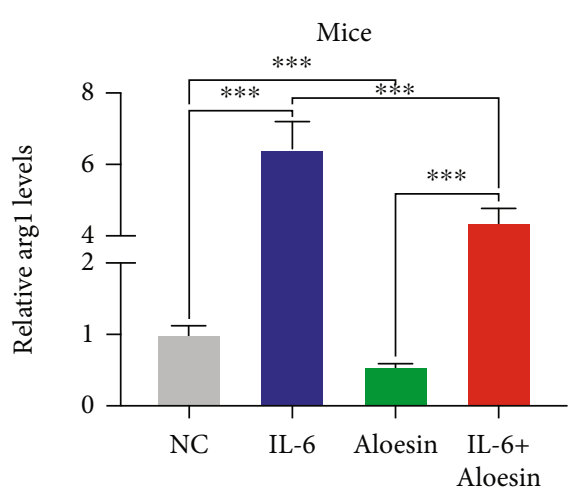

(e)

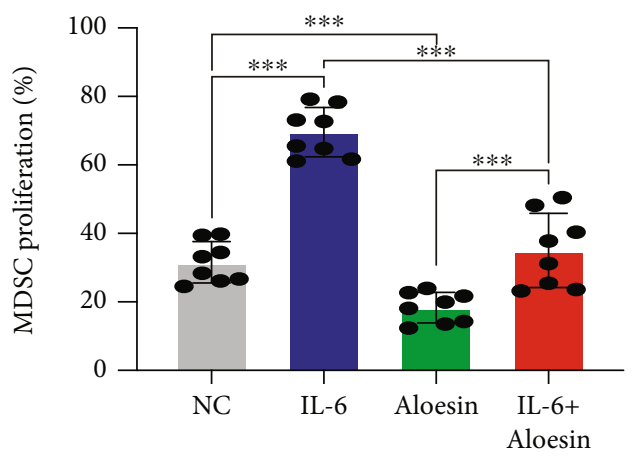

(b)

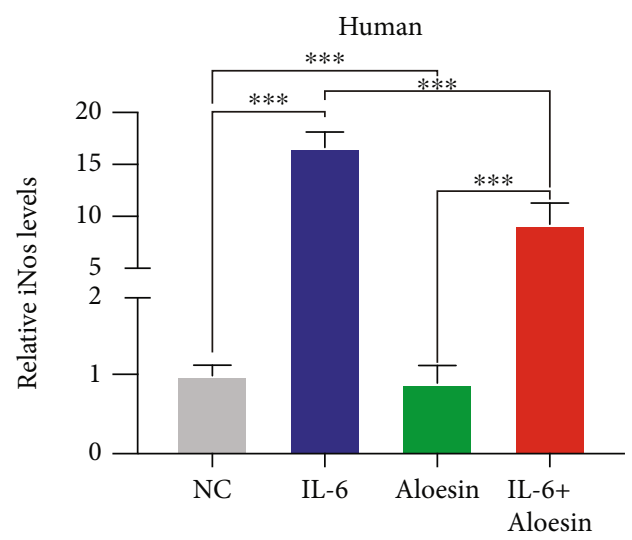

(d)

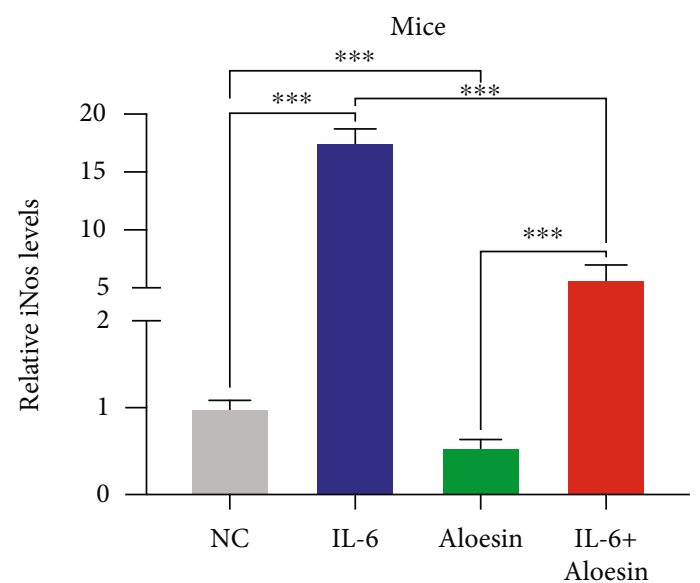

(f)

FIgure 6: Continued. 


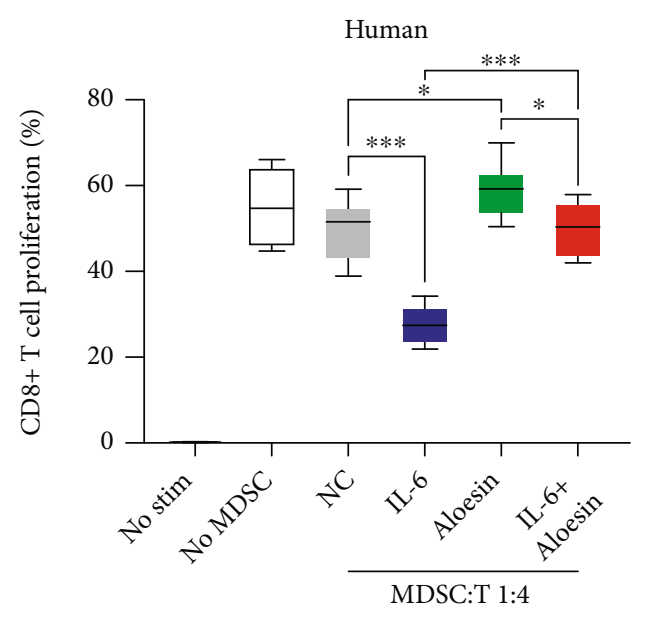

(g)

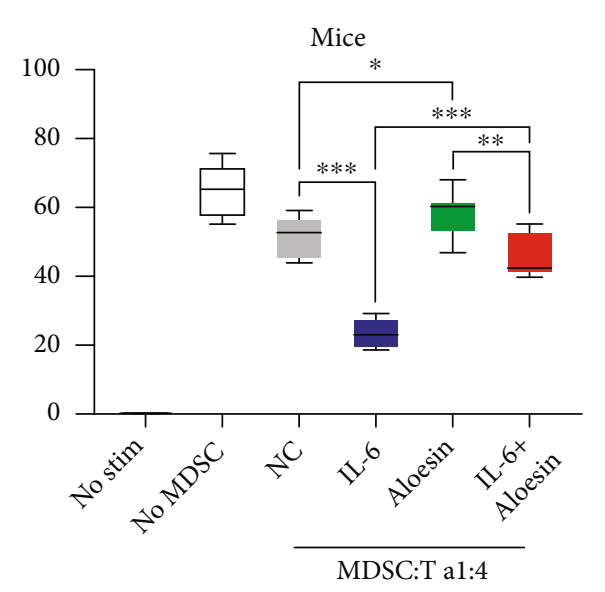

(i)

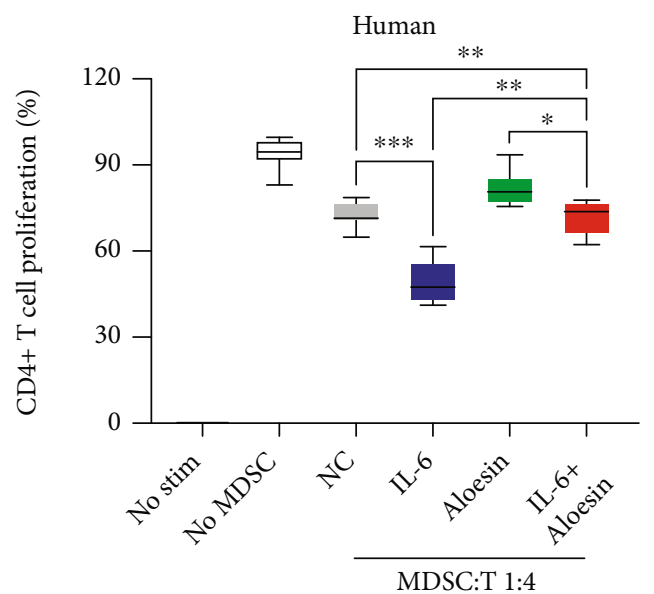

(h)

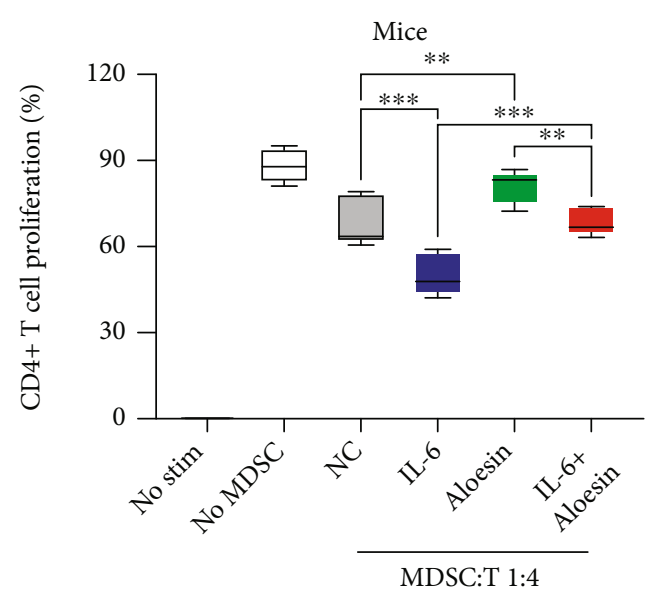

(j)

FIGURE 6: IL-6 promoted MDSC proliferation and immune-suppressive effects by activating the MAPK signaling pathway. (a) CCK-8 assays were used to evaluate MDSCs with or without IL-6 and/or aloesin treatment. (b) MDSC proliferation rates were evaluated according to fluorescence attenuation after IL-6 and/or aloesin treatment. (c-f) RT-PCR was used to evaluate Arg1 and iNOS levels in MDSCs with or without IL-6 and/or aloesin treatment. (g-j) Summary of the suppressive effect of MDSCs by coculture with CD ${ }^{+} \mathrm{T}^{\mathrm{c}}$ cells and CD $4^{+} \mathrm{T}$ cells at $1: 4$ ratios. Mean $\pm \mathrm{SD},{ }^{*} P<0.05,{ }^{* *} P<0.01$, and ${ }^{* * *} P<0.005$.

with patients with low frequencies of these myeloid cells [33]. Peripheral MDSCs have a high correlation with tumor progression and grade, and low MDSC levels in circulation are significantly associated with an improved prognosis and outcomes in BC [34].

This study investigated the mechanisms by which IL-6 activated MDSCs, identifying the important role of the MAPK signaling pathway in MDSC proliferation in MIBC. Nevertheless, the precise interactions between IL-6 and MAPK are still unclear. Bongartz et al. reported that IL6-induced MAPK activation could be categorized into a Gab1-independent early phase and a Gab1-dependent late phase. Gab1-independent MAPK activation in the early phase is vital for the follow-up launch of the Gab1dependent expansion of MAPK activation, with the binding of the IL-6 receptor complex and SH2 domaincontaining phosphatase 2 (SHP2). The successive synergetic recruitment of Grb2 and SHP2 to Gab1 is crucial for the Gab1-dependent augmentation of IL-6, eliciting MAPK signaling pathway activation in the late phase and the upregulation of various genes [35]. Additionally, MAPK cascade activation caused by IL-6 relies critically on the recruitment of SHP2 to Y759 of phosphorylation in the cytoplasmic territory of gp13063 [36].

ERK, a member of the MAPK family, is a survivalpromoting factor in immunocytes, indicating that its activity may harbor immune-stimulating or immunesuppressive effects relying on the cell types penetrating the solid tumor [37]. ERK can activate the amplification of $\mathrm{CD}^{+}$TILs and promote the secretion of lytic granules and cytokines, upgrading the cytotoxicity of TILs in the TME [38]. The critical role of immature MDSCs in immune evasion was mediated by ERK in the present study. Generally, a subset of MDSCs shared numerous similarities with TAM; however, the upregulation of the MAPK pathway could polarize macrophages from the 


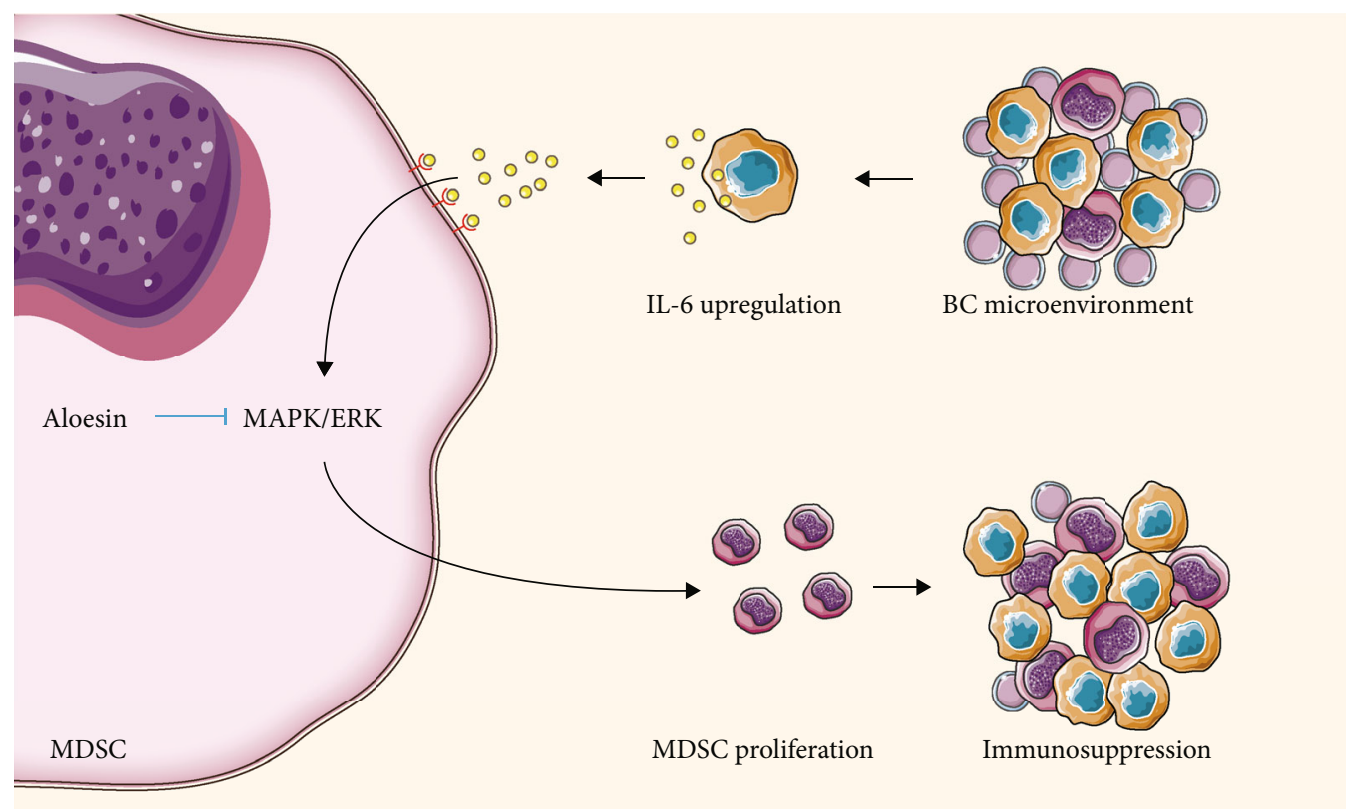

FIGURE 7: Schematic model. IL-6 promotes the proliferation and immunosuppressive function of MDSCs via the MAPK signaling pathway in MIBC.

M2 phenotype to M1 phenotype and lead to the inhibition of the suppressive effects of MDSCs by the stimulation of LPS [39]. STAT3 inhibition could decrease MAPK and lead to the apoptosis of MDSCs [40]. In BC, tumor cells induced MDSC aggregation and expansion in the TME via the CXCL2/MIF-CXCR2 signaling pathway and the elevated phosphorylation of p38, ERK, and p65 [41].

In summary, the data suggested that IL- 6 promoted MIBC progression by not only accelerating proliferation but also improving the immune suppression ability of MDSCs via activating the MAPK signaling pathway.

\section{Abbreviations}

BC: $\quad$ Bladder cancer

NMIBC: Non-muscle-invasive bladder cancer

MIBC: Muscle-invasive bladder cancer

TIL: $\quad$ Tumor-infiltrating lymphocyte

TME: Tumor microenvironment

MDSC: Myeloid-derived suppressor cell

IL-6: $\quad$ Interleukin 6

DC: Dendritic cell

MAPK: Mitogen-activated protein kinase

ERK: $\quad$ Extracellular signal-regulated kinase

ELISA: Enzyme-linked immunosorbent assay

CFSE: Carboxyfluorescein diacetate succinimidyl ester

RT-PCR: Reverse transcription-polymerase chain reaction

HPRT: Hypoxanthine phosphoribosyltransferase

SD: $\quad$ Standard deviation

CCK-8: Cell Counting Kit-8

ARG1: $\quad$ Arginase 1

iNOS: Inducible nitric oxide synthase

MEK2: $\quad$ Mitogen-activated protein kinase 2

MNK1: Mitogen-activated protein kinase-interacting kinase 1
PI3K: Phosphoinositide 3-kinase

AKT: $\quad$ Protein kinase B

Stat3: $\quad$ Signal transducer and activator of transcription 3

Gab1: $\quad$ GRB2-associated-binding protein 1

SHP2: $\quad$ SH2 domain-containing phosphatase 2

LPS: Lipopolysaccharide

CXCL2: C-X-C motif chemokine ligand 2

CXCR2: C-X-C chemokine receptor 2

AOD: Average optical density

MFI: Median fluorescence intensity.

\section{Data Availability}

The detailed procedures of methods and figures are attached. The data used to support the findings of this study are available from the corresponding author upon request.

\section{Additional Points}

Précis. IL-6 strengthens the immunosuppressive ability of MDSCs via the MAPK signaling pathway in MIBC.

\section{Ethical Approval}

This work was approved by the Ethical Committee of Shanghai Jiao Tong University according to the Chinese Ethical Regulations. All animal experiments complied with the Shanghai Jiao Tong University of Medicine Policy on the Care and Use of Laboratory Animals.

\section{Consent}

The authors confirmed that we have obtained written consent from the patients to publish the manuscript. 


\section{Disclosure}

The funders had no role in the design and conduct of the study; collection, management, analysis, and interpretation of the data; preparation, review, or approval of the manuscript; and decision to submit the manuscript for publication.

\section{Conflicts of Interest}

The authors declare that they have no competing interests.

\section{Authors' Contributions}

Junhua Zheng was responsible for the conceptualization and funding acquisition. Ke Wu was responsible for the supervision and resources. Zhihong Liu was responsible for the project administration and funding acquisition. Zhong Zheng was responsible for the methodology, software, and validation. Xinyi Zheng was responsible for the formal analysis and investigation. Yiwen Zhu was responsible for the data curation and visualization. Zhixian Yao was responsible for the software and writing of the original draft. Weiguang Zhao was involved in the writing of the original draft. Youjia Zhu was involved in the review and editing of the manuscript. Feng Sun was involved in the review and editing of the manuscript. Xingyu Mu was involved in the writing of the original draft. Yong Wang was involved in the writing of the original draft. Wanqing He was involved in the review and editing of the manuscript. Zhong Zheng, Xinyi Zheng, and Yiwen Zhu contributed equally to this work.

\section{Acknowledgments}

We acknowledge the support from the Youth Science and Technology Innovation Studio of Shanghai Jiao Tong University School of Medicine. The reported work was supported in part by research grants from the National Natural Science Foundation of China (nos. 81972393, 81772705, 31570775, 81672515, and 82072823), Shanghai Municipal Education Commission-Gaofeng Clinical Medicine Grant (20172019), and the Natural Science Foundation of Shanghai (20ZR1444900).

\section{Supplementary Materials}

Table S1: monoclonal antibodies used for flow cytometric assay. Table S2: antibodies used for western blotting. Table S3: statistics of the RNA-seq data for human MDSCs. Table S4: statistics of the RNA-seq data for mice MDSCs. (Supplementary Materials)

\section{References}

[1] N. Mahdavifar, M. Ghoncheh, R. Pakzad, Z. Momenimovahed, and H. Salehiniya, "Epidemiology, incidence and mortality of bladder cancer and their relationship with the development index in the world," Asian Pacific Journal of Cancer Prevention, vol. 17, no. 1, pp. 381-386, 2016.
[2] L. Klotz and M. A. Brausi, "World Urologic Oncology Federation Bladder Cancer Prevention Program: a global initiative," Urologic Oncology, vol. 33, no. 1, pp. 25-29, 2015.

[3] S. Lei, H. Xu, N. Chen et al., "MKP-1 overexpression is associated with chemoresistance in bladder cancer via the MAPK pathway," Oncology Letters, vol. 20, no. 2, pp. 1743-1751, 2020.

[4] P. L. Crispen and S. Kusmartsev, "Mechanisms of immune evasion in bladder cancer," Cancer Immunology, Immunotherapy, vol. 69, no. 1, pp. 3-14, 2020.

[5] A. K. Schneider, M. F. Chevalier, and L. Derré, "The multifaceted immune regulation of bladder cancer," Nature Reviews. Urology, vol. 16, no. 10, pp. 613-630, 2019.

[6] W. H. Fridman, F. Pagès, C. Sautès-Fridman, and J. Galon, "The immune contexture in human tumours: impact on clinical outcome," Nature Reviews. Cancer, vol. 12, no. 4, pp. 298-306, 2012.

[7] Z. Zheng, S. Mao, W. Zhang et al., "Dysregulation of the immune microenvironment contributes to malignant progression and has prognostic value in bladder cancer," Frontiers in Oncology, vol. 10, article 542492, 2020.

[8] D. I. Gabrilovich, "Myeloid-derived suppressor cells," Cancer Immunology Research, vol. 5, no. 1, pp. 3-8, 2017.

[9] D. M. Pardoll, "The blockade of immune checkpoints in cancer immunotherapy," Nature Reviews. Cancer, vol. 12, no. 4, pp. 252-264, 2012.

[10] J. B. Lamano, J. B. Lamano, Y. D. Li et al., "Glioblastomaderived IL6 induces immunosuppressive peripheral myeloid cell PD-L1 and promotes tumor growth," Clinical Cancer Research, vol. 25, no. 12, pp. 3643-3657, 2019.

[11] T. A. Karakasheva, E. W. Lin, Q. Tang et al., "IL-6 mediates cross-talk between tumor cells and activated fibroblasts in the tumor microenvironment," Cancer Research, vol. 78, no. 17, pp. 4957-4970, 2018.

[12] M. L. Ibrahim, C. Lu, J. D. Klement et al., "Expression profiles and function of IL6 in polymorphonuclear myeloid-derived suppressor cells," Cancer Immunology, Immunotherapy, vol. 69, no. 11, pp. 2233-2245, 2020.

[13] J. S. C. Arthur and S. C. Ley, "Mitogen-activated protein kinases in innate immunity," Nature Reviews Immunology, vol. 13, no. 9, pp. 679-692, 2013.

[14] E. K. Kim and E.-J. Choi, "Pathological roles of MAPK signaling pathways in human diseases," Biochimica et Biophysica Acta (BBA) - Molecular Basis of Disease, vol. 1802, no. 4, pp. 396-405, 2010.

[15] X. Y. Mu, R. J. Wang, Z. X. Yao et al., "RS 504393 inhibits MMDSCs recruiting in immune microenvironment of bladder cancer after gemcitabine treatment," Molecular Immunology, vol. 109, pp. 140-148, 2019.

[16] C. Garbers, H. M. Hermanns, F. Schaper et al., "Plasticity and cross-talk of interleukin 6-type cytokines," Cytokine \& Growth Factor Reviews, vol. 23, no. 3, pp. 85-97, 2012.

[17] D. E. Johnson, R. A. O'Keefe, and J. R. Grandis, "Targeting the IL-6/JAK/STAT3 signalling axis in cancer," Nature Reviews. Clinical Oncology, vol. 15, no. 4, pp. 234-248, 2018.

[18] K. Taniguchi and M. Karin, "IL-6 and related cytokines as the critical lynchpins between inflammation and cancer," Seminars in Immunology, vol. 26, no. 1, pp. 54-74, 2014.

[19] S. A. Jones and B. J. Jenkins, "Recent insights into targeting the IL-6 cytokine family in inflammatory diseases and cancer," 
Nature Reviews. Immunology, vol. 18, no. 12, pp. 773-789, 2018.

[20] M. F. Chen, P. Y. Lin, C. F. Wu, W. C. Chen, and C. T. Wu, "IL-6 expression regulates tumorigenicity and correlates with prognosis in bladder cancer," PLoS One, vol. 8, no. 4, article e61901, 2013.

[21] H. Wei, "Interleukin 6 signaling maintains the stem-like properties of bladder cancer stem cells," Translational Cancer Research, vol. 8, no. 2, pp. 557-566, 2019.

[22] D. B. Thompson, L. E. Siref, M. P. Feloney, R. J. Hauke, and D. K. Agrawal, "Immunological basis in the pathogenesis and treatment of bladder cancer," Expert Review of Clinical Immunology, vol. 11, no. 2, pp. 265-279, 2015.

[23] M. R. Cardillo, P. Sale, and F. Di Silverio, "Heat shock protein90, IL-6 and IL-10 in bladder cancer," Anticancer Research, vol. 20, no. 6b, pp. 4579-4583, 2000.

[24] N. Kumari, U. Agrawal, A. K. Mishra et al., "Predictive role of serum and urinary cytokines in invasion and recurrence of bladder cancer," Tumour Biology, vol. 39, no. 4, article 101042831769755, 2017.

[25] B. Andrews, S. F. Shariat, J. H. Kim, T. M. Wheeler, K. M. Slawin, and S. P. Lerner, "Preoperative plasma levels of interleukin-6 and its soluble receptor predict disease recurrence and survival of patients with bladder cancer," The Journal of Urology, vol. 167, no. 3, pp. 1475-1481, 2002.

[26] H. Tsukamoto, K. Fujieda, S. Senju, T. Ikeda, H. Oshiumi, and Y. Nishimura, "Immune-suppressive effects of interleukin-6 on T-cell-mediated anti-tumor immunity," Cancer Science, vol. 109, no. 3, pp. 523-530, 2018.

[27] M. Jiang, J. Chen, W. Zhanget al. et al., "Interleukin-6 TransSignaling Pathway Promotes Immunosuppressive MyeloidDerived Suppressor Cells via Suppression of Suppressor of Cytokine Signaling 3 in Breast Cancer," Frontiers in Immunology, vol. 8, p. 1840, 2017.

[28] H. L. Young, E. J. Rowling, M. Bugatti et al., “An adaptive signaling network in melanoma inflammatory niches confers tolerance to MAPK signaling inhibition," The Journal of Experimental Medicine, vol. 214, no. 6, pp. 1691-1710, 2017.

[29] F. Y. Mao, Y. L. Zhao, Y. P. Lv et al., "CD45 $\mathrm{CD} 33^{\text {low }} \mathrm{CD} 11 \mathrm{~b}^{\text {dim }}$ myeloid-derived suppressor cells suppress $\mathrm{CD}^{+} \mathrm{T}$ cell activity via the IL-6/IL-8-arginase I axis in human gastric cancer," Cell Death \& Disease, vol. 9, no. 7, p. 763, 2018.

[30] M. Xu, Z. Zhao, J. Song et al., "Interactions between interleukin- 6 and myeloid-derived suppressor cells drive the chemoresistant phenotype of hepatocellular cancer," Experimental Cell Research, vol. 351, no. 2, pp. 142-149, 2017.

[31] G. Yang, W. Shen, Y. Zhang et al., "Accumulation of myeloidderived suppressor cells (MDSCs) induced by low levels of IL6 correlates with poor prognosis in bladder cancer," Oncotarget, vol. 8, no. 24, pp. 38378-38388, 2017.

[32] D. Marvel and D. I. Gabrilovich, "Myeloid-derived suppressor cells in the tumor microenvironment: expect the unexpected," The Journal of Clinical Investigation, vol. 125, no. 9, pp. 33563364, 2015.

[33] R. P. Tobin, D. Davis, K. R. Jordan, and M. D. McCarter, “The clinical evidence for targeting human myeloid-derived suppressor cells in cancer patients," Journal of Leukocyte Biology, vol. 102, no. 2, pp. 381-391, 2017.

[34] E. Eruslanov, M. Neuberger, I. Daurkin et al., "Circulating and tumor-infiltrating myeloid cell subsets in patients with bladder cancer," International Journal of Cancer, vol. 130, no. 5, pp. 1109-1119, 2012.

[35] H. Bongartz, K. Gille, W. Hessenkemper et al., "The multi-site docking protein Grb2-associated binder 1 (Gab1) enhances interleukin-6-induced MAPK-pathway activation in an SHP2-, Grb2-, and time-dependent manner," Cell Communication and Signaling: CCS, vol. 17, no. 1, p. 135, 2019.

[36] W. P. Schiemann, J. L. Bartoe, and N. M. Nathanson, "Box 3independent Signaling Mechanisms Are Involved in Leukemia Inhibitory Factor Receptor $\alpha \backslash$ - and gp130-mediated Stimulation of Mitogen-activated Protein Kinase:," The Journal of Biological Chemistry, vol. 272, no. 26, pp. 16631-16636, 1997.

[37] I. C. Salaroglio, E. Mungo, E. Gazzano, J. Kopecka, and C. Riganti, "ERK is a pivotal player of chemo-immuneresistance in cancer," International Journal of Molecular Sciences, vol. 20, no. 10, p. 2505, 2019.

[38] L. K. Robertson, L. R. Mireau, and H. L. Ostergaard, “A role for phosphatidylinositol 3-kinase in TCR-stimulated ERK activation leading to paxillin phosphorylation and CTL degranulation," Journal of Immunology, vol. 175, no. 12, pp. 8138-8145, 2005.

[39] Y. Yang, R. Zhang, F. Xia et al., "LPS converts Gr- $1^{+} \mathrm{CD} 115^{+}$ myeloid- derived suppressor cells from M2 to M1 via P38 MAPK," Experimental Cell Research, vol. 319, no. 12, pp. 1774-1783, 2013.

[40] P. Guha, J. Gardell, J. Darpolor et al., "STAT3 inhibition induces Bax-dependent apoptosis in liver tumor myeloidderived suppressor cells," Oncogene, vol. 38 , no. 4, pp. 533548, 2019.

[41] H. Zhang, Y. L. Ye, M. X. Li et al., "CXCL2/MIF-CXCR2 signaling promotes the recruitment of myeloid-derived suppressor cells and is correlated with prognosis in bladder cancer," Oncogene, vol. 36, no. 15, pp. 2095-2104, 2017. 